\title{
Inhibition of IRAK1/4 sensitizes T cell acute lymphoblastic leukemia to chemotherapies
}

\author{
Zhaoyang Li, ${ }^{1}$ Kenisha Younger, ${ }^{2}$ Ronald Gartenhaus, ${ }^{2,3}$ Ann Mary Joseph, ${ }^{2}$ Fang Hu, ${ }^{2}$ Maria R. Baer, ${ }^{2,3}$ Patrick Brown, ${ }^{4}$ \\ and Eduardo Davila ${ }^{2,5}$ \\ 'Children's National Medical Center, Center for Cancer and Immunology Research, Washington DC, USA. ${ }^{2}$ University of Maryland Marlene and Stewart Greenebaum Cancer Center, Baltimore, Maryland, USA. \\ ${ }^{3}$ Department of Medicine, University of Maryland School of Medicine, Baltimore, Maryland, USA. ${ }^{2}$ Departments of Oncology and Pediatrics, Sidney Kimmel Comprehensive Cancer Center and Johns Hopkins \\ University School of Medicine, Baltimore, Maryland, USA. D.pepartment of Microbiology and Immunology, University of Maryland School of Medicine, Baltimore, Maryland, USA.
}

\begin{abstract}
Signaling via the MyD88/IRAK pathway in T cells is indispensable for cell survival; however, it is not known whether this pathway functions in the progression of T acute lymphoblastic leukemia (T-ALL). Here, we determined that compared with thymic and peripheral T cells, T-ALL cells from patients have elevated levels of IRAK1 and IRAK4 mRNA as well as increased total and phosphorylated protein. Targeted inhibition of IRAK1 and IRAK4, either with shRNA or with a pharmacological IRAK1/4 inhibitor, dramatically impeded proliferation of T-ALL cells isolated from patients and T-ALL cells in a murine leukemia model; however, IRAK1/4 inhibition had little effect on cell death. We screened several hundred FDA-approved compounds and identified a set of drugs that had enhanced cytotoxic activity when combined with IRAK inhibition. Administration of an IRAK1/4 inhibitor or IRAK knockdown in combination with either ABT-737 or vincristine markedly reduced leukemia burden in mice and prolonged survival. IRAK1/4 signaling activated the E3 ubiquitin ligase TRAF6, increasing K63-linked ubiquitination and enhancing stability of the antiapoptotic protein MCL1; therefore, IRAK inhibition reduced MCL1 stability and sensitized T-ALL to combination therapy. These studies demonstrate that IRAK1/4 signaling promotes T-ALL progression through stabilization of MCL1 and suggest that impeding this pathway has potential as a therapeutic strategy to enhance chemotherapeutic efficacy.
\end{abstract}

\section{Introduction}

Acute lymphoblastic leukemia (ALL) accounts for approximately one-third of cancers in children (0-19 years of age), making it the most common cancer in this age group (1-3). $\mathrm{T}$ cell ALL (T-ALL) represents $10 \%-15 \%$ of ALL cases in children and $25 \%$ of adult T-ALL cases. The use of conventional cancer therapies has resulted in a complete remission rate of $85 \%$ and a high cure rate in childhood T-ALL, but adult T-ALL patients are at increased risk of both early BM recurrence and CNS relapse. The prognosis for relapsing patients is poor, with only $15 \%-25 \%$ achieving stable remission after second-line treatment (1-3), and the 5-year survival rate for adult T-ALL patients is only $45 \%-55 \%$. These outcomes underscore the need to develop more effective therapies to treat T-ALL patients.

Recent studies highlight an indispensable role for MyD88 signaling in primary T cells (4-10). The engagement of IL-1 receptor family members as well as TLRs (except TLR3) recruits the adapter protein MyD88, which in turn brings in an IL-1 receptor-associated kinase 4 (IRAK4), resulting in autophosphorylation. IRAK4 recruits and phosphorylates IRAK1. Activated IRAK1 binds to and activates TNF receptor-associated factor 6 (TRAF6). Depending on the cell type on which IRAK4/1 signaling occurs, it can result in the activation of various transcription factors including NF- $\mathrm{B}, \mathrm{AP}-1, \mathrm{CREB}$,

Conflict of interest: The authors have declared that no conflict of interest exists Submitted: February 24, 2014; Accepted: December 11, 2014.

Reference information: / Clin Invest. 2015;125(3):1081-1097. doi:10.1172/JCI75821. and IRF5 that ultimately promote cell survival or proliferation (11-13). TRAF6 is an E3 ubiquitin ligase and catalyzes K63 polyubiquitination of TAK1, which is required for IKK activation and is known to directly regulate ubiquitination and activation of AKT and mTORC1 as well as TGF- $\beta$ (14-17). Interestingly, CD4 or CD8 $\mathrm{T}$ cells lacking MyD88 exhibit reduced expansion and impaired survival in vivo (4-10). IRAK4 has been reported to be recruited to T cell lipid rafts, where it associates with ZAP70 and participates in protein kinase $\mathrm{C}$ activation (18). T cells from patients with IRAK4 or MyD88 deficiency exhibit defects in activation and proliferation, highlighting a critical role for IRAK4 signaling in T cell activation and survival $(19,20)$. Furthermore, studies by several groups, including ours, have demonstrated that activating MyD88/IRAK signaling via TLR engagement on CD4 Th cells or CD8 T cells substantially enhances proliferation $(5,21-25)$. Engagement of TLRs has also been shown to prolong cell survival, which correlates with increased expression levels of BCL-xL and BCL2 $(26,27)$, as well as $\mathrm{A} 1$, and reduced levels of $\operatorname{BIM}(24,26,27)$.

Given the prominent role that the MyD88/IRAK4 signaling axis plays in primary $\mathrm{T}$ cell survival and considering its emerging role as a contributor to the progression of various hematologic malignancies (28-31), the goal of this study was to gain a greater understanding of the role of IRAK1/4 signaling in the growth and survival of T cell neoplasms. We found that T-ALL cells expressed elevated levels of IRAK1 and IRAK4 mRNA as well as increased levels of total and activated (phosphorylated) IRAK1 and IRAK4. Inhibition of IRAK4 using shRNA or a small-molecule inhibitor 
impeded cell proliferation and, perhaps more importantly, augmented the cytotoxic effects of various molecularly targeted and chemotherapeutic agents, including ABT-737 and vincristine. These synergistic effects were in large part dependent on MCL1. At a mechanistic level, IRAK4 signaling regulates MCL1 expression levels by increasing its biosynthesis and enhancing protein stability (but not by increasing transcription). IRAK1/4 signaling activates TRAF6, an E3 ubiquitin ligase, which correlated with K63linked MCL1 ubiquitination and enhanced MCL1 protein stability. The biological significance of targeting IRAK4 signaling in T-ALL was highlighted by demonstrating that treatment with IRAK1/4 inhibitor suppressed T-ALL expansion in xenograft models and, more importantly, that combination therapy with IRAK1/4 inhibitor and ABT-737 or vincristine considerably reduced T-ALL burden in mice and prolonged their survival. Our study highlights a previously uncharacterized and critical role for IRAK4 signaling in T-ALL proliferation and chemoresistance and indicates that IRAK signaling may have a pathophysiological role and clinical implications for patients with T-ALL and other T cell malignancies.

\section{Results}

IRAK1 and IRAK4 are overexpressed and activated in T-ALL. We evaluated IRAK1 and IRAK4 mRNA levels in 917 tumor cell lines, comparing 15 T-ALL cell lines and 902 other cell lines of different tumor types (Figure 1A) (32). We found that IRAK4 transcript levels were overexpressed in T-ALL in relation to various other tumor cells and to thymic T cell controls. Increased IRAK4 levels were comparable to those in other hematological malignancies including B cell acute lymphoblastic leukemia (B-ALL), acute myeloid leukemia (AML), and chronic lymphocytic leukemia (CLL) (Figure $1 \mathrm{~A}$ ) and were among the highest in the entire set of cell lines studied, including diverse solid tumors (Figure 1A). On the other hand, IRAK1 mRNA levels in T-ALL cell lines were comparable to those in other tumor types examined (Figure 1A).

We examined IRAK1 and IRAK4 protein levels in several malignant $\mathrm{T}$ cell lines. All lines except MOLT4 expressed variably high levels of IRAK1 and IRAK4 (Figure 1B). IRAK4 kinase activity is regulated by autophosphorylation at threonine 345 (Thr345) and serine 346 (Ser346) (12, 33-35), which, upon phosphorylation, activates IRAK1 or in some cases IRAK2 (36). To determine the activation status of IRAK1 and IRAK4, we examined the phosphorylation levels of IRAK1 (Thr209) and IRAK4 (Thr345/Ser346) by Western blotting $(12,13)$. As shown in Figure 1B, phosphorylated IRAK1 (Thr209) [p-IRAK1 (Thr209)] was detected in 9 of 10 lines. IRAK4 was highly phosphorylated in most lines. Because IRAK2 can also associate with IRAK4 and substitute for IRAK1 in some cell types (36), its expression levels were also examined and found to be weak and variable among the different cell lines. We also studied IRAK4 and IRAK1 expression levels in solid tumors and found that, while some tumors expressed IRAK1 and IRAK4, their phosphorylation levels were lower (or absent) compared with those of T-ALL cells (Supplemental Figure 1C; supplemental material available online with this article; doi:10.1172/JCI75821DS1). We found both IRAK4 and IRAK1 proteins to be highly expressed in the cytoplasm of CCRF-CEM and Jurkat T-ALL cells (Figure 1C), as determined by immunofluorescence (IF) staining. Furthermore, in agreement with the Western blot findings, IRAK4, but not IRAK1, was detected by IF in MOLT4 cells.
IRAK1 and IRAK4 mRNA levels were also elevated in T-ALL cells from patients compared with the levels detected in thymic $\mathrm{T}$ cells or $\mathrm{T}$ cells from peripheral blood (Figure 1D and Supplemental Figure 2A). The expression levels of other pattern recognition receptor-related genes were found to be altered in T-ALL cells compared with those in thymic T cell controls (Supplemental Figure 2B). Furthermore, consistent with the increased transcript levels in malignant $\mathrm{T}$ cell lines, patient samples showed higher IRAK4 and IRAK1 protein levels than did peripheral blood mononuclear cells (PBMCs) (Figure 1E). In fact, p-IRAK4 and p-IRAK1 were only detected in T-ALL samples. We found that purified $\mathrm{T}$ cells from healthy donors expressed very low levels of IRAK1 and IRAK4, and what little protein we detected was unphosphorylated (Supplemental Figure 1A). IRAK2 was detected, and its levels were comparable among all samples. It is worth noting that the cell-selection process did not induce the phosphorylation of IRAK1 or IRAK 4 and that IRAK expression in primary T cells was only evident upon TCR stimulation (Supplemental Figure 1D and 1F). Collectively, these data highlight that IRAK1 and IRAK4 are overexpressed and activated in T-ALL.

IRAK1/4 signaling inhibition impedes T-ALL proliferation. To determine whether IRAK1/IRAK4 signaling contributes to T-ALL proliferation, we inhibited IRAK1/4 activity using a small-molecule inhibitor that selectively inhibits the kinase activities of IRAK1 and IRAK4 (37). As shown in Figure 2A, IRAK1/4 inhibition significantly impaired the proliferation of 8 of 10 malignant $\mathrm{T}$ cell lines in a concentration-dependent manner. It is worth noting that IRAK1/4 inhibition did not impede MOLT4 proliferation, which correlates with the lack of IRAK1 expression. Similarly, IRAK1/4 inhibition reduced proliferation in 4 of $5 \mathrm{~T}$-ALL patient samples (Figure 2B). The effects of the IRAK1/4 inhibitor on IRAK signaling were validated by its ability to reduce the phosphorylation of IRAK1 (Thr209) and IRAK4 (Thr345/Ser346), which did not affect overall protein levels (Figure $2 \mathrm{C}$ ). That the antiproliferative effects occurred in an IRAK1/4dependent manner was confirmed by knocking down IRAK1 or IRAK4 expression through transient transfection using shRNAs (Figure 2D; $P<0.05$ ); IRAK1- and IRAK4-knockdown efficiency is shown in Figure 2D. Likewise, the lack of IRAK4 kinase activity in T cells reduced their ability to proliferate (Supplemental Figure 1E). We also examined IRAK2 expression levels as controls and found that they were not affected by expression of either IRAK1 or IRAK 4 shRNA.

TLR1/TLR2 signaling in T-ALL can augment proliferation. Recent studies demonstrated that engagement of TLR1/TLR2 on primary $\mathrm{T}$ cells enhances their proliferation and survival (5, 21-25). We sought to determine whether the TLR1-TLR2 ligand, which activates IRAK4 signaling, could enhance T-ALL proliferation. Addition of the synthetic TLR1-TLR2 agonist Pam3CysK4 increased IRAK1 and IRAK4 activation and proliferation in CCRFCEM cells (Figure 3A and Supplemental Figure 3A). However, we found that blocking TLR2 signaling with neutralizing anti-TLR2 antibody or IRAK1/4 inhibitor diminished Pam3CysK4-induced proliferation, confirming that these effects occurred by a TLR2IRAK-dependent mechanism (Figure 3, A and B). It is important to note that while inhibiting IRAK1/4 signaling reduced basal T-ALL proliferation, blocking TLR2 alone did not alter proliferation. These data indicate that TLR2 is not necessary for T-ALL cells to proliferate and that IRAK4 signaling plays an important role in 
A
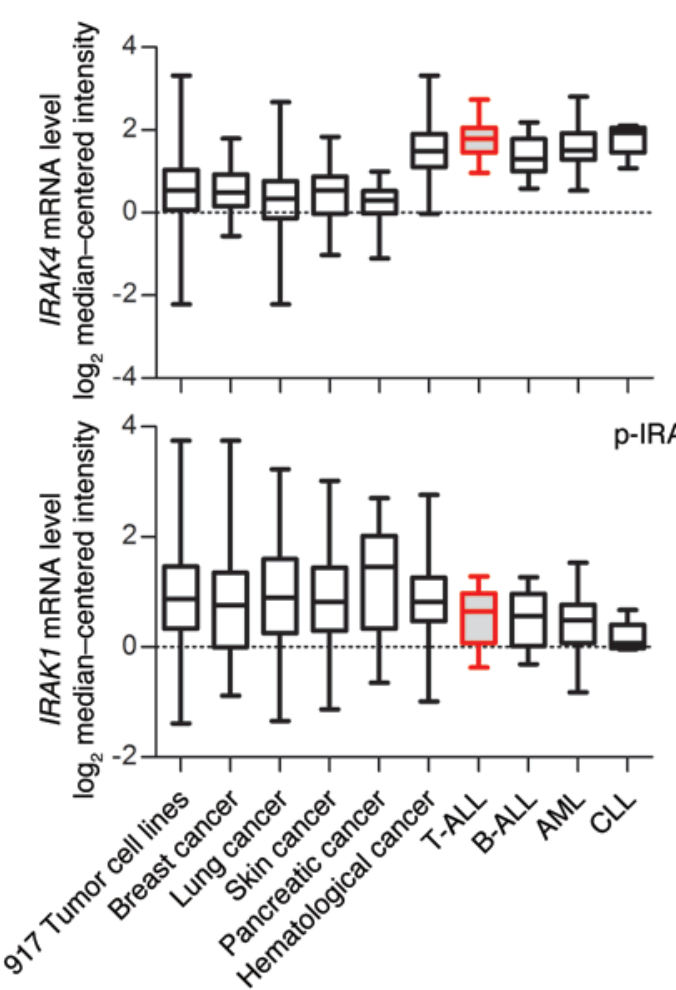

D IRAK4

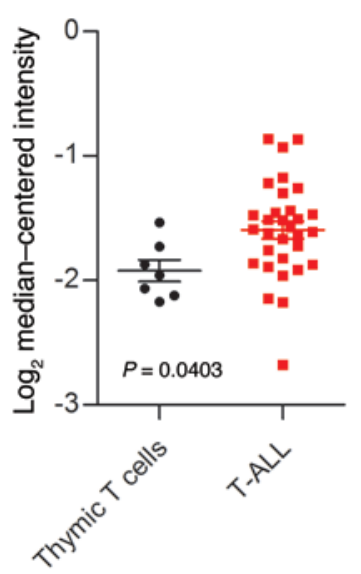

IRAK1

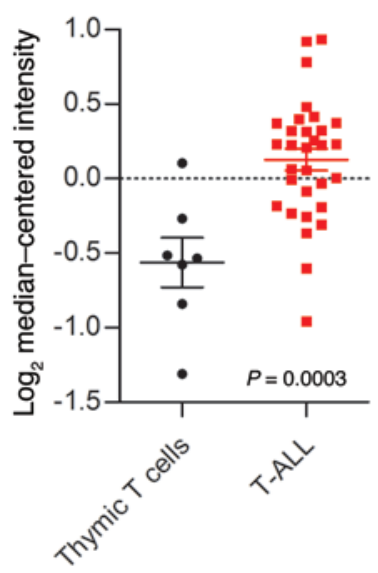

B

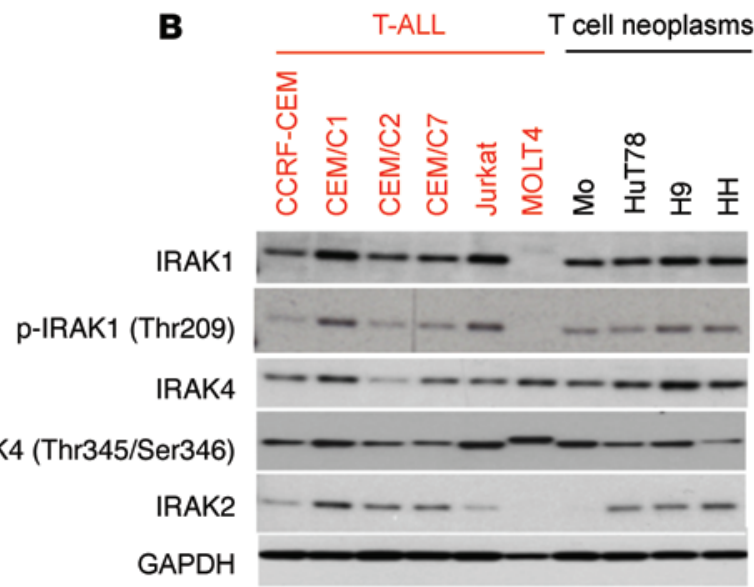

C
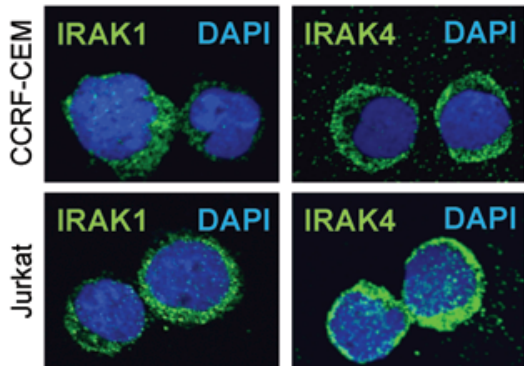

IRAK4 DAPI
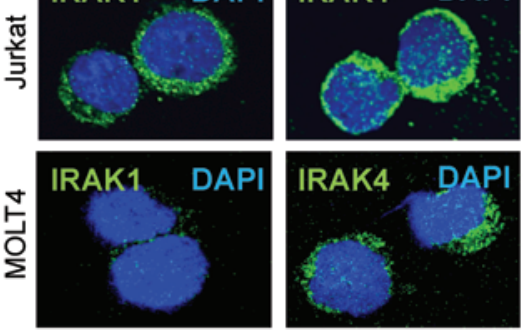

E

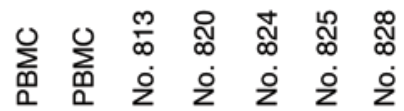

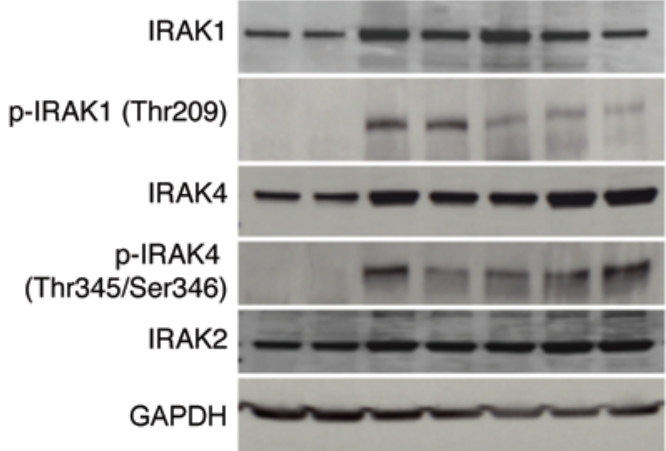

Figure 1. IRAK1 and IRAK4 expression levels and activation status in T-ALL. (A) Microarray (GEO GSE36133) analysis of IRAK4 and IRAK1 gene expression levels in 917 tumor cell lines, grouped as indicated. Lines, bars, and whiskers represent the median, quartiles, and minimum and maximum values, respectively. (B) Expression levels of the indicated proteins in various T-ALL lines and other neoplastic T cell lines were determined by Western blot analysis. GAPDH served as a loading control. (C) Confocal microscopic images showing IF staining of IRAK1 and IRAK4 protein in CCRF-CEM, Jurkat, and MOLT4 cells (original magnification, $\times 40$ ). (D) Microarray analysis (CEO GSE46170) of IRAK1 and IRAK4 mRNA levels in sorted thymic T cells $(n=7)$ and patient peripheral T-ALL $(n=31)$ cells. Each symbol represents 1 patient, and whiskers represent the median and quartile values. $P<0.05$ by Student's $t$ test. (E) Total or p-IRAK1 and p-IRAK4 and IRAK2 protein levels in PBMCs, as well as patient T-ALL cells, were examined by Western blotting. GAPDH served as a loading control.

T-ALL proliferation. We also examined the proliferative effects of TLR1-TLR2 agonists in 5 T-ALL patient samples and found that 2 of the 5 samples showed increased proliferation in response to TLR1-TLR2 ligand (Figure 3C; $P<0.01$ at concentrations above $1.25 \mu \mathrm{g} / \mathrm{ml}$ ). Importantly, the 3 samples (nos. 824, 825, and 828) that did not respond to the TLR1-TLR2 agonist expressed similar levels of TLR1, TLR2, and MyD88 (Supplemental Figure 1B), but exhibited much higher basal proliferation compared with that observed in the 2 samples that responded to TLR1-TLR2 ligand (nos. 813 and 820). These data indicate that the lack of response to 
TLR agonists was not due to a lack of TLR or MyD88 expression. However, it is important to note that despite their lack of response to TLR1-TLR2 ligand, T-ALL cells were strongly inhibited by the IRAK1/4 inhibitor (Figure 2B). To explore in greater detail whether other TLR agonists influence the proliferation of malignant T cells, we examined the effects of TLR1 through TLR9 agonists in 14 human malignant $\mathrm{T}$ cell lines (Supplemental Table 1). In general, we found that the TLR1-TLR2 agonist promoted the proliferation of T-ALL lines, whereas most of the other TLR agonists we tested had little to no effect, and reduced proliferation in some cases.

We further examined whether TLR1-TLR2 agonists could pro-

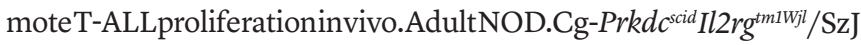
IL2RG (NSG) mice were injected i.v. with CCRF-CEM cells, followed by injection with TLR1-TLR2 ligand. We determined the frequency of CCRF-CEM T-ALL cells using anti-human CD7 and anti-human CD5 antibodies after normalizing samples with calibration beads, as shown in Figure 3D and Supplemental Figure 4A. Administration of TLR1-TLR2 ligand increased T-ALL cell numbers in the BM and peripheral blood compared with those in control mice (Figure 3D; $P<0.05)$. The results demonstrate that TLR2 ligand can promote leukemia progression in vivo and suggest that activating IRAK signaling via TLR stimulation on T-ALL cells could contribute to T-ALL progression.

In vivo treatment with IRAK1/4 inhibitor reduces T-ALL cell numbers and delays $T$-ALL progression. We examined the therapeutic efficacy of IRAK1/4 inhibitor in mice with T-ALL. The data in Figure 4A demonstrate that treatment with IRAK1/4 inhibitor significantly reduced the percentage and total number of $\mathrm{hCD}^{+}$ CCRF-CEM T-ALL cells in the BM and circulation compared with treatment with DMSO vehicle or no treatment (Figure 4A, $P<0.05$, and Supplemental Figure 4B). To further validate that impairment of IRAK1/4 signaling in T-ALL impedes expansion in vivo, T-ALL cells were stably transduced with lentiviral vector encoding the shRNA targeting IRAK1 (which is downstream of IRAK4) or control lentiviral vector ( $\mathrm{pGIPZ}$ vector containing GFP). We selected Jurkat T-ALL cells, since they responded to the IRAK inhibitor and were more amenable to lentiviral transduction than were CCRF-CEM cells. Stably transduced $\mathrm{GFP}^{+}$cells were sorted and i.v. injected into NSG mice, and 21 days later, the number of T-ALL cells in the BM and circulation was determined by flow cytometry. The data in Figure $4 \mathrm{~B}$ and Supplemental Figure 4, C and D, demonstrate that knocking down IRAK1 decreased cell numbers in the BM, peripheral blood, and spleen. These data underscore the finding that impeding IRAK signaling within T-ALL cells alters growth in vivo. We further examined whether treatment with IRAK1/4 inhibitor reduced expansion of patient-derived T-ALL samples. T-ALL samples were allowed to engraft into NSG mice until approximately 100 T-ALL cells per microliter of blood were detected by flow cytometry, and the mice were then treated with IRAK1/4 inhibitor. Figure $4 \mathrm{C}$ shows a representative dot plot indicating a similar number of T-ALL cells in peripheral blood on day 7, just prior to treatment. However, on day 21, the number of T-ALL cells was markedly reduced in mice treated with IRAK1/4 inhibitor compared with that detected in control mice (day 21) or in samples collected from the same mice on day 7. In general, IRAK1/4 inhibitor treatment reduced T-ALL cell numbers (from 3 different patients) compared with the num- bers detected in DMSO-treated control mice (Figure 4C; $P<0.01$ ). Furthermore, treatment with IRAK1/4 inhibitor significantly prolonged mouse survival (Figure $4 \mathrm{D} ; P=0.02$ ). Collectively, these data indicate that targeting IRAK1/4 signaling in T-ALL impairs leukemia progression and prolongs host survival.

Identification of therapeutic compounds that synergize with IRAK1/4 inhibitor. In light of the ability of IRAK1/4 inhibitor to suppress T-ALL proliferation in vitro and reduce its accumulation in vivo, we examined its effects on apoptosis. We found that IRAK1/4 inhibitor suppressed T-ALL proliferation and maintained cell numbers at steady state over the course of 96 hours in vitro (Supplemental Figure 5, A and B). However, the reduced proliferation was not the result of apoptosis, as examined by propidium iodide (PI) and annexin V staining (Supplemental Figure 5C). Furthermore, the lack of cell death was not due to insufficient concentrations of the IRAK inhibitor, as increasing concentrations had little effect on cell death, and the effects leveled off at $10 \mu \mathrm{M}$ (Supplemental Figure 5D). Cell-cycle analyses showed that the percentage of cells in the GO/G1 phase increased from $25 \%$ to $43 \%$ within 24 hours of adding IRAK1/4 inhibitor and increased further to $50 \%$ after 96 hours (Supplemental Figure 5E). These results indicate that IRAK1/4 inhibitor suppresses T-ALL proliferation through delayed cell-cycle entry, but has minimal effect on cell viability.

Considering that IRAK inhibition moderately reduced T-ALL expansion in vivo and did not significantly influence apoptosis, we evaluated whether combining IRAK1/4 inhibitor with other therapeutic agents could potentiate their cytotoxic effect in vitro. We screened 484 FDA-approved compounds at various concentrations together with IRAK1/4 inhibitor or DMSO for 48 hours, at which time the effects were determined by examining cell proliferation. This high-throughput screening generated approximately 22 hits, with the vast majority belonging to 4 groups of inhibitors: EGFR inhibitors, inhibitors and disruptors of tubulin, HSP9O inhibitors, and BCL2 family inhibitors (Figure 5A). Among these 4 groups, a tubulin inhibitor, vincristine, has long been used for clinical treatment of ALL patients, and BCL2 family inhibitors are currently being evaluated in phase I and II clinical trials for leukemia (https://www.clinicaltrials.gov; NCT00481091 and NCT00868413). We proceeded to validate the effects of vincristine and ABT-737 by examining apoptosis of CCRF-CEM cells with each of the chemotherapy drugs at various concentrations plus IRAK1/4 inhibitor. IRAK1/4 inhibitor augmented the cytotoxic activities of both ABT-737 and vincristine in a synergistic fashion (Figure 5B); we verified the synergy between compounds using the Chou-Talalay method of analysis (Figure 5B). We observed similar effects in the Jurkat T-ALL cell line (Figure $5 \mathrm{C}$ and Supplemental Figure 6B). To further confirm that the synergistic effects occurred in an IRAK signaling-dependent fashion, IRAK1 or IRAK4 were knocked down with shRNA, and cells were cultured with ABT-737 at various concentrations. We found that knocking down either IRAK1 or IRAK4 protein significantly enhanced ABT-737-induced apoptosis (Figure 5E; $P<0.05$ ). Combinatorial therapy was also more effective and functioned in a synergistic fashion in T-ALL patient samples (Figure 5D and Supplemental Figure 6, A and C). Collectively, these results indicate that impeding IRAK1/4 signaling in T-ALL significantly augments the cytotoxic effects of various therapeutic agents. 

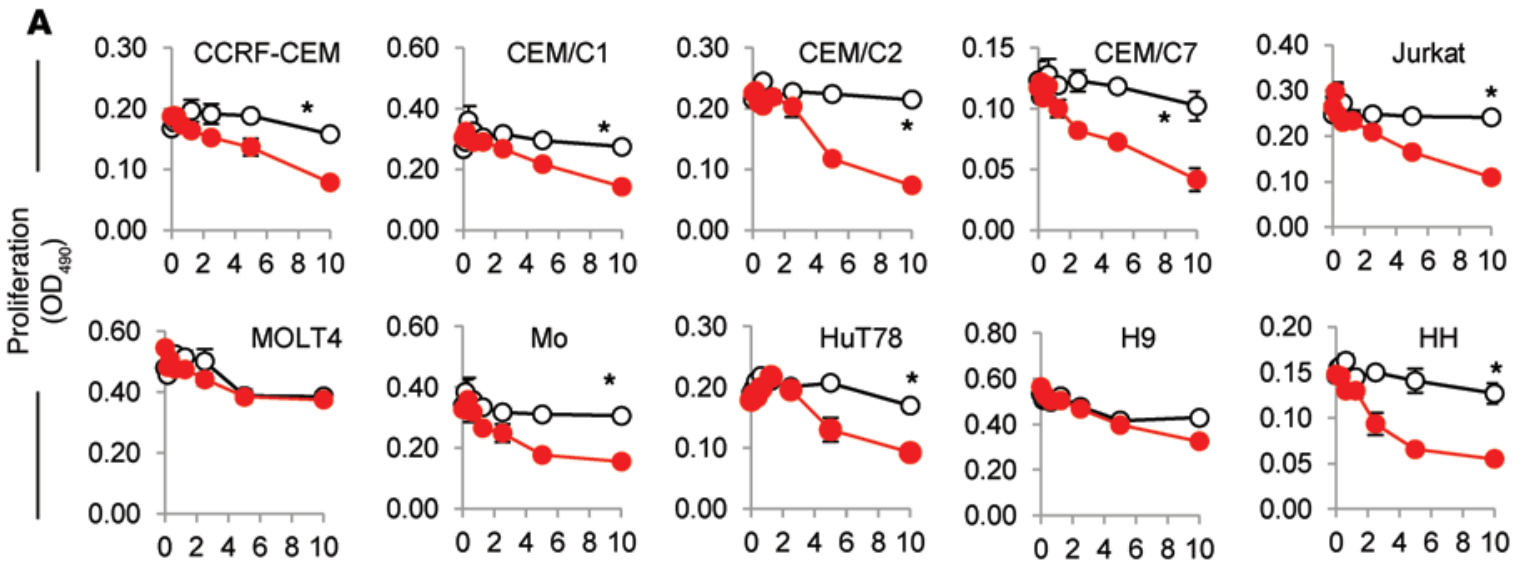

O DMSO

IRAK $1 / 4$
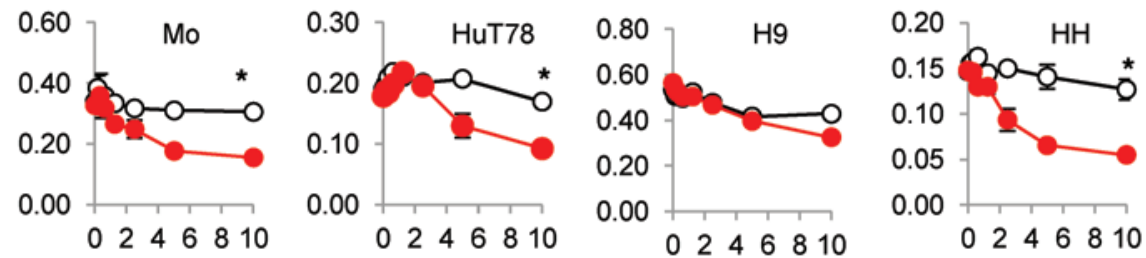

IRAK1/4 inhibitor $(\mu \mathrm{M})$
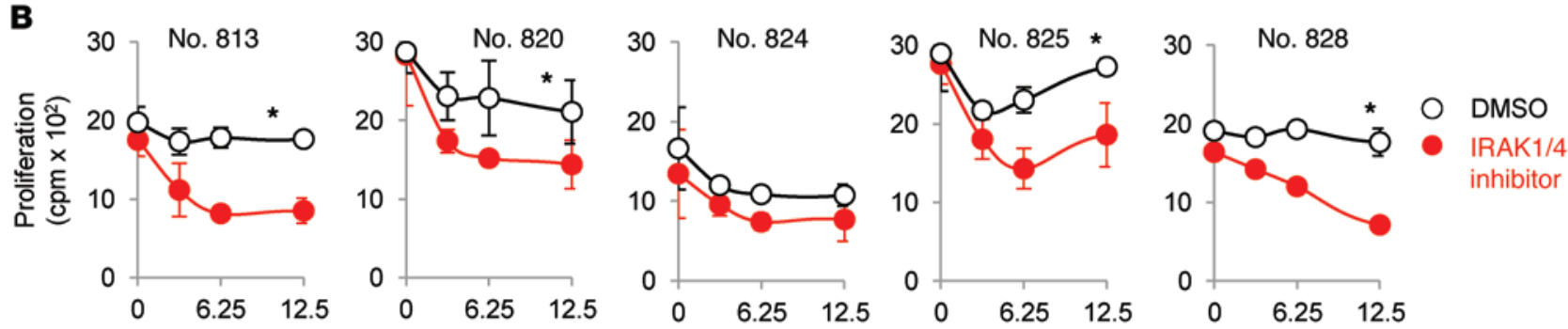

C

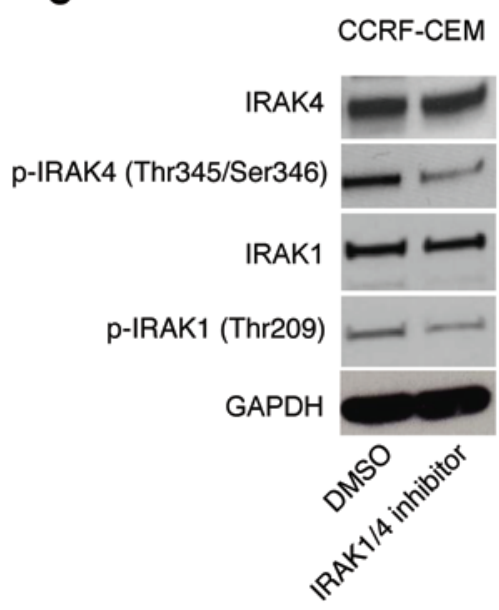

D

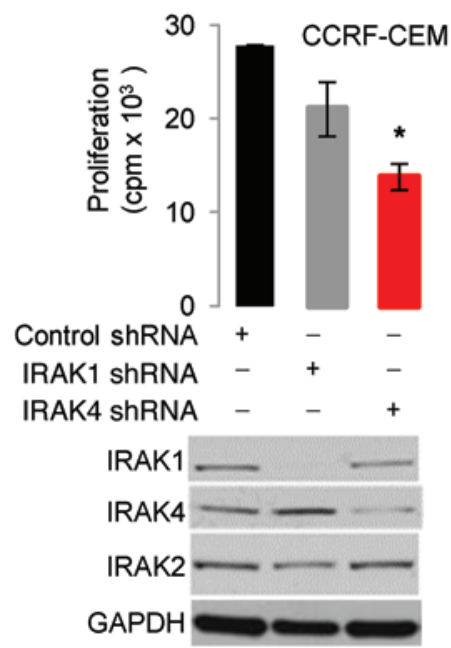

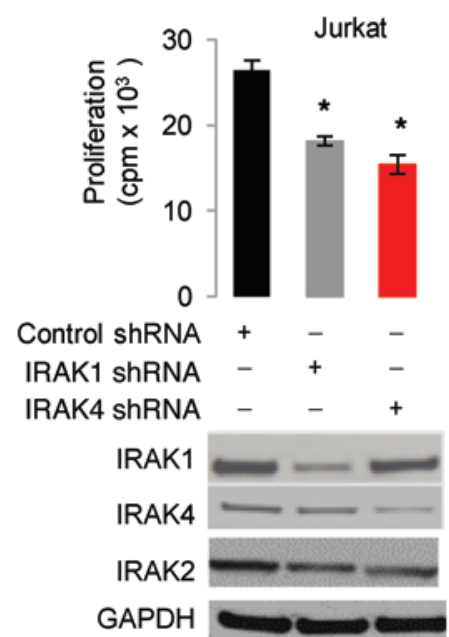

Figure 2. IRAK1/4 signaling inhibition impedes T-ALL proliferation. (A) IRAK4/1 inhibitor effects on the proliferation of various malignant T cell lines. Cells were cultured in the presence of IRAK1/4 inhibitor (0-10 $\mu \mathrm{M})$ or control (DMSO), and proliferation was measured 72 hours later. Data are representative of 3 independent experiments, and the average $\mathrm{OD}_{490}( \pm \mathrm{SD})$ of triplicate readings is shown. (B) Patient T-ALL cells were cultured in the presence of control (DMSO) or IRAK1/4 inhibitor $(0-12.5 \mu \mathrm{M})$, and proliferation was determined by measuring ${ }^{3}[\mathrm{H}]$ thymidine incorporation. Data represent the average of triplicate readings ( \pm SD) and are representative of 2 independent experiments, each yielding identical trends. ${ }^{*} P<0.05$ by Student's $t$ test (A and B). (C) Levels of the indicated proteins were examined in CCRF-CEM T-ALL cells after treatment with IRAK1/4 inhibitor (2.5 $\mu$ M) or DMSO for 48 hours. (D) T-ALL cells were transiently transfected with the indicated plasmids. Transfected cells were sorted by flow cytometry according to GFP expression, and proliferation of CCRF-CEM and Jurkat cells was determined according to ${ }^{3}[\mathrm{H}]$ thymidine uptake (top panels). Average cpm ( \pm SD) of triplicate readings is shown. ${ }^{*} P<0.05$ versus control shRNA by Student's $t$ test. Knockdown efficiency was examined by Western blotting. Data are representative of 3 independent experiments. 
A

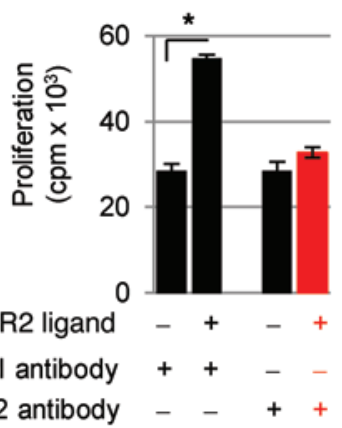

c

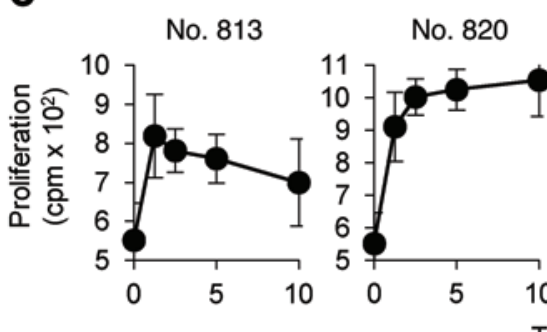

No. 813
B

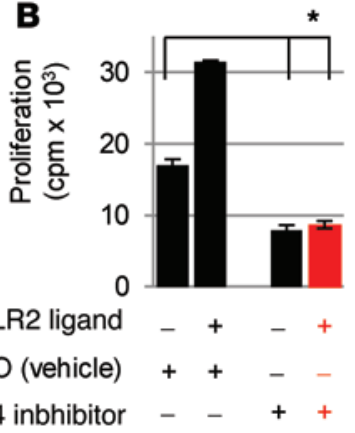

T-ALL patient samples

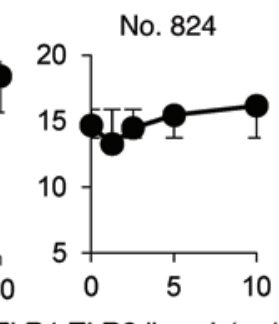

D

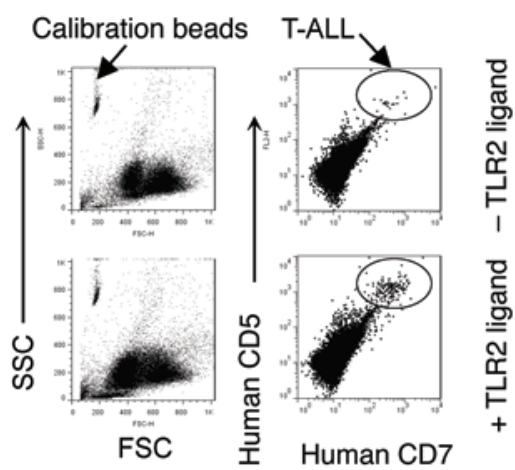

$\square-$ TLR1-TLR2 ligand

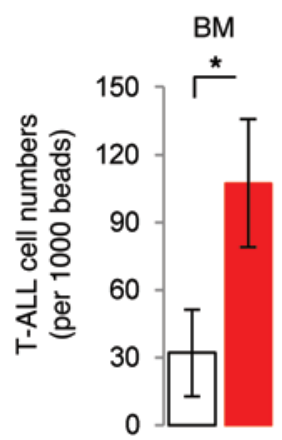

Figure 3. TLR1-TLR2 engagement can augment T-ALL proliferation. (A) CCRF-CEM cells were treated with $\mathrm{Pam}_{3} \mathrm{CysK}_{4}$ $(2.5 \mu \mathrm{g} / \mathrm{ml})$ or control (PBS) in the presence or absence of either $5 \mathrm{mg} / \mathrm{ml}$ neutralizing anti-TLR2 antibody or isotype control antibody. After 48 hours, proliferation was determined by ${ }^{3}[\mathrm{H}]$ thymidine incorporation. (B) CCRF-CEM cells were cultured with DMSO or IRAK1/4 inhibitor $(1.5 \mu \mathrm{M})$ in the presence or absence of Pam $_{3}$ CysK $_{4}(2.5 \mu \mathrm{g} / \mathrm{ml})$, and after 48 hours, proliferation was determined by ${ }^{3}[\mathrm{H}]$ thymidine incorporation. Data in $\mathbf{A}$ and $\mathbf{B}$ are representative of 3 independent experiments, and the average cpm ( \pm SD) of triplicate readings is shown. ${ }^{*} P<0.05$ by Student's $t$ test. (C) Patient T-ALL samples were treated with the indicated concentrations of $\mathrm{Pam}_{3} \mathrm{CysK}_{4}$ for 48 hours. Proliferation was determined by ${ }^{3}[\mathrm{H}]$ thymidine incorporation, and data are representative of 2 experiments. (D) CCRF-CEM cells $\left(3 \times 10^{6}\right)$ were i.v. injected into NSG mice $(n=5)$. Seven day later, the mice were injected with $\mathrm{Pam}_{3} \mathrm{CysK}_{4}(2 \mathrm{mg} / \mathrm{kg})$ or control (PBS), and the number of T-ALL cells in circulation and BM was determined on day 14 by staining cells with anti-human CD7 and CD5 antibodies and analyzed by flow cytometry. Cell counts were normalized by adding an equal volume of calibration beads to $50 \mu \mathrm{l}$ blood and setting the instrument gates to count a constant number of beads. Representative dot plots (left panel) and absolute numbers in BM and blood (right panel). Data represent the average cell counts ( \pm SD) in 5 mice and are representative of 2 independent experiments. ${ }^{*} P<0.05$ by Student's $t$ test. FSC, forward scatter; SSC, side scatter.

Combination treatment with IRAK1/4 inhibitor and ABT-737 or vincristine reduces T-ALL burden and prolongs host survival. NSG mice with established T-ALL were treated with IRAK1/4 inhibitor or ABT-737 using suboptimal doses of each. As shown in Figure 6, A and B, treatment with IRAK1/4 inhibitor or ABT-737 alone moderately reduced T-ALL burden, and the percentage and absolute count of T-ALL cells were similar to those in control-treated mice. In sharp contrast, com-

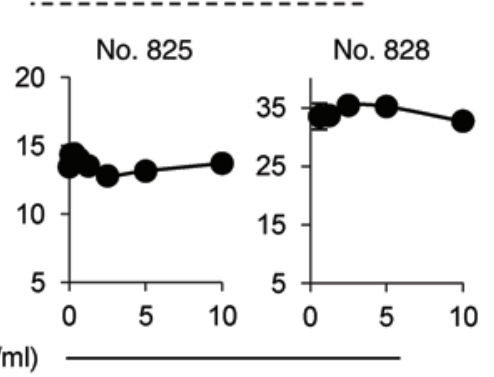

+ TLR1-TLR2 ligand

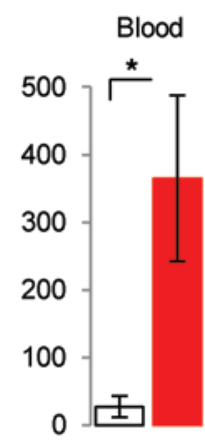

bination treatment with IRAK1/4 inhibitor and ABT-737 significantly reduced T-ALL cell counts in $\mathrm{BM}$ and peripheral blood (Figure 6, A and B, and Supplemental Figure 7A). Consistent with the in vitro data shown in Figure 5, coadministration of IRAK1/4 inhibitor and vincristine decreased T-ALL burden in mice (Figure 6C and Supplemental Figure 7B). To confirm that the observed effects were mediated by altering IRAK signaling within T-ALL cells, and not as a result of altering IRAK signaling in other cell types, mice were injected with T-ALL cells engineered to stably express shRNA IRAK1 (or control vector) and treated or not treated with ABT-737. As shown in Figure $6 \mathrm{D}$, administration of ABT-737 considerably reduced leukemia cell counts in BM and peripheral blood (Supplemental Figure 7C). The combinatorial effects of these 2 drugs were visually pronounced when examining the spleens (Figure $6 \mathrm{E}$ and Supplemental Figure 7D). It is worth noting that knocking down IRAK was sufficient to reduce T-ALL expansion and was as effective as treating mice with ABT-737, highlighting the important role of IRAK signaling within T-ALL cells in leukemia progression.

Coadministration ofIRAK1/4 inhibitor and ABT-737 significantly prolonged mouse survival (median survival, 40 days) as compared with survival of DMSO-treated control mice (median survival, 27 days) (Figure 6F). Treatment with IRAK1/4 inhibitor or ABT-737 alone was statistically inferior to treatment with IRAK1/4 inhibitor plus ABT-737 (Figure 6F). The power values are provided in Figure $6 \mathrm{~F}$. A caveat to conducting studies in xenogeneic models is that mice have a severely compromised immune system, and how therapy might impact immune responses or vice versa is not known. We examined the effects that this treatment regimen had on different immune cell subsets in WT C57BL/6 mice and detected no significant changes in the percentages of $\mathrm{B}$ cells, $\mathrm{CD} 4^{+}$or $\mathrm{CD} 8^{+}$ $\mathrm{T}$ cells, dendritic cells, macrophages, NK cells, or BM stem cells (Supplemental Figure 8). 
A
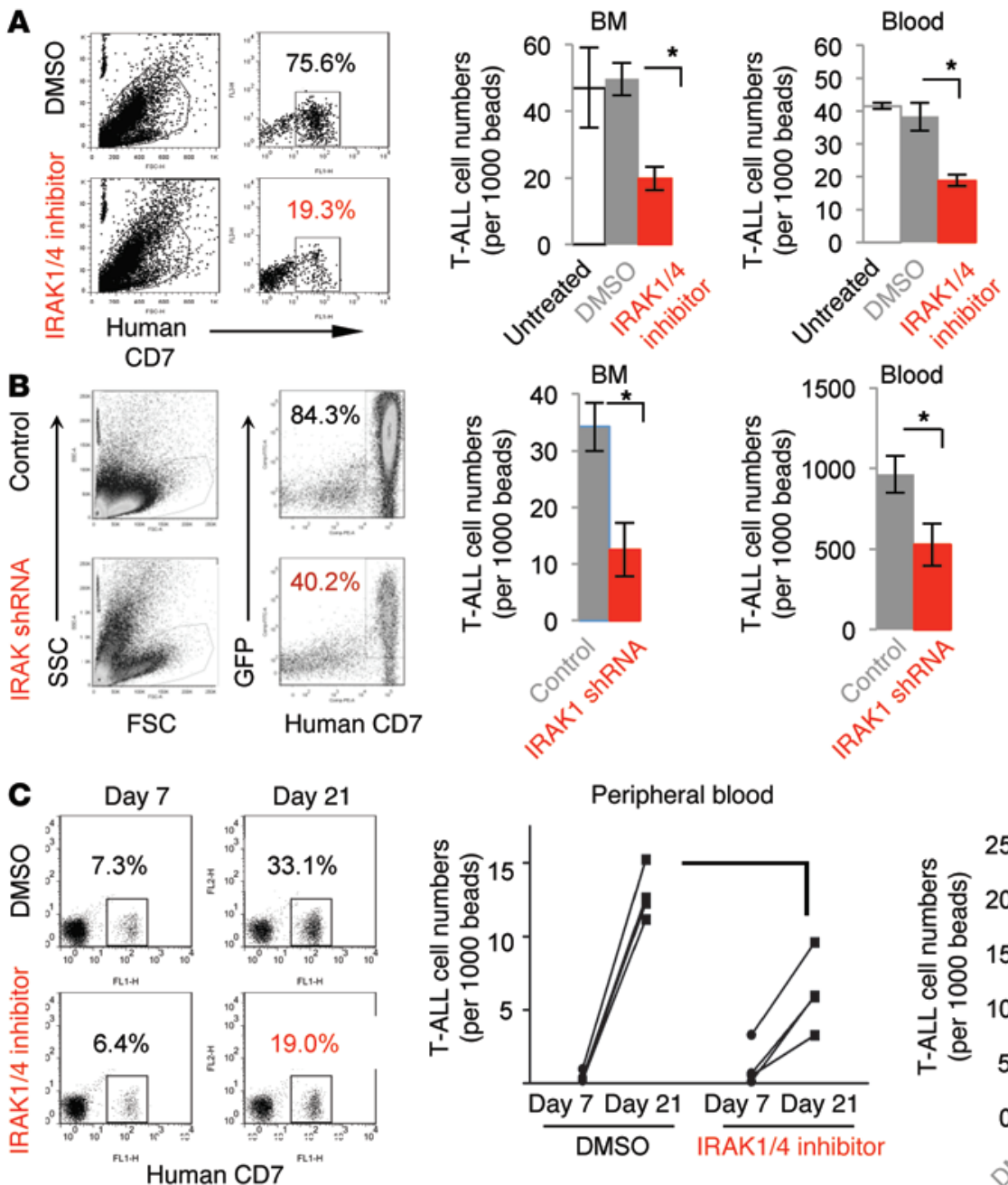

D

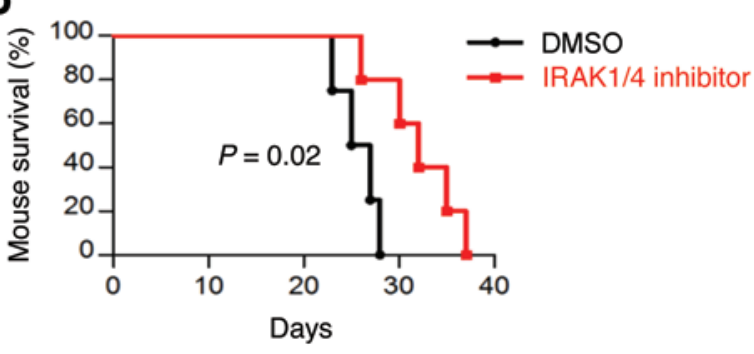

Figure 4. In vivo intervention with IRAK1/4 inhibitor reduces T-ALL numbers and delays T-ALL progression. (A) CCRF-CEM cells were injected i.v. into NSC mice, followed by i.p. injection with IRAK1/4 inhibitor $(10 \mathrm{mg} / \mathrm{kg}$ ) or DMSO on days 3, 6, and 9. Numbers of T-ALL cells (distinguished by CD7 expression) in BM and blood were determined by flow cytometry on day 21. Representative flow cytometric dot plots and average number of cells from 5 mice $( \pm S D)$ are shown. (B) Jurkat cell lines with reduced IRAK1 expression were generated using lentiviral vectors and injected i.v. into NSG mice. On day 21, the number of T-ALL cells in BM and blood were measured by flow cytometry. (C) NSG mice were injected i.v. with T-ALL cells from 3 different patients. Mice with equal numbers of T-ALL cells in the blood were identified by flow cytometry on day 7 (dot plot) and injected i.p. with IRAK1/4 inhibitor or DMSO on days 7, 10, and 13. Numbers represent the percentages of T-ALL cells. T-ALL cells were enumerated in blood and BM on day 21 and are shown per 100,000 beads in the bar graphs. (D) NSG mice were injected with CCRF-CEM cells and treated with IRAK1/4 inhibitor or DMSO on days 3, 6, and 9. (A, B and D) Data are representative of 2 independent experiments ( 5 mice/group) and were analyzed by Student's $t$ test (A and B), ANOVA (C), and log-rank test (D).
A previous study comparing refractory patients with those who responded to chemotherapy found IL-8 to be highly expressed in refractory T-ALL (38). Given that inhibition of IRAK signaling dramatically enhanced the effects of chemotherapeutic drugs, we sought to determine whether there was an association between IRAK1 transcript levels and response to chemotherapy. Using 2 independent gene expression array data sets, we compared baseline (pretreatment) IRAK1 expression levels in patients who achieved prolonged remission with expression levels in those who relapsed, but found no apparent differences (Supplemental Figure 2C). Unfortunately, IRAK4 was not included in these original gene expression arrays, and its potential role in chemoresistance or relapse could therefore not be investigated.

IRAK1/4 inhibition alters the expression of key molecules associated with cell survival and proliferation and protein synthesis. To gain a mechanistic understanding of how IRAK inhibition in T-ALL impacts the cell cycle and survival, we compared protein expression profiles between IRAK1/4 inhibitor-treated and control (DMSO-treated) cells using a focused cell-cycle and apoptosis antibody array. The scatter plot in Supplemental Figure 9A shows the changes in protein expression levels among the samples. We validated the changes in some of these proteins by Western blot analysis. In particular, we found a significant reduction in the levels of $\mathrm{p}-\mathrm{p} 65$, p-mTOR, p-AKT, and MCL1 following IRAK1/4 inhibition (Figure 7A). In contrast, IRAK inhibition did not alter p-ERK1/2 levels, nor did it have an effect on BCL2 or BCL-xL (Figure 7A and Supplemental Figure 9F). The ability of IRAK inhibition to reduce mTOR phosphorylation and activity is in agreement with previous reports indicating that stimulating TLR1/TLR2 signaling (which activates IRAK1/4) profoundly activates AKT signaling and mTOR activity in T cells $(6,8)$. 
A

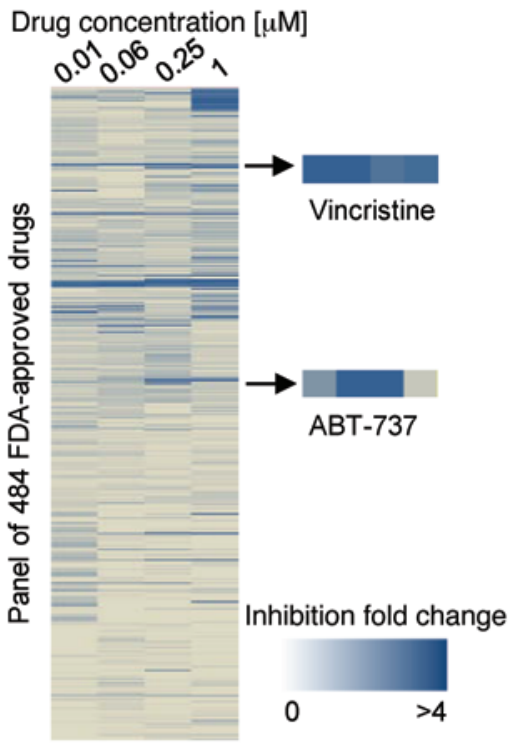

B
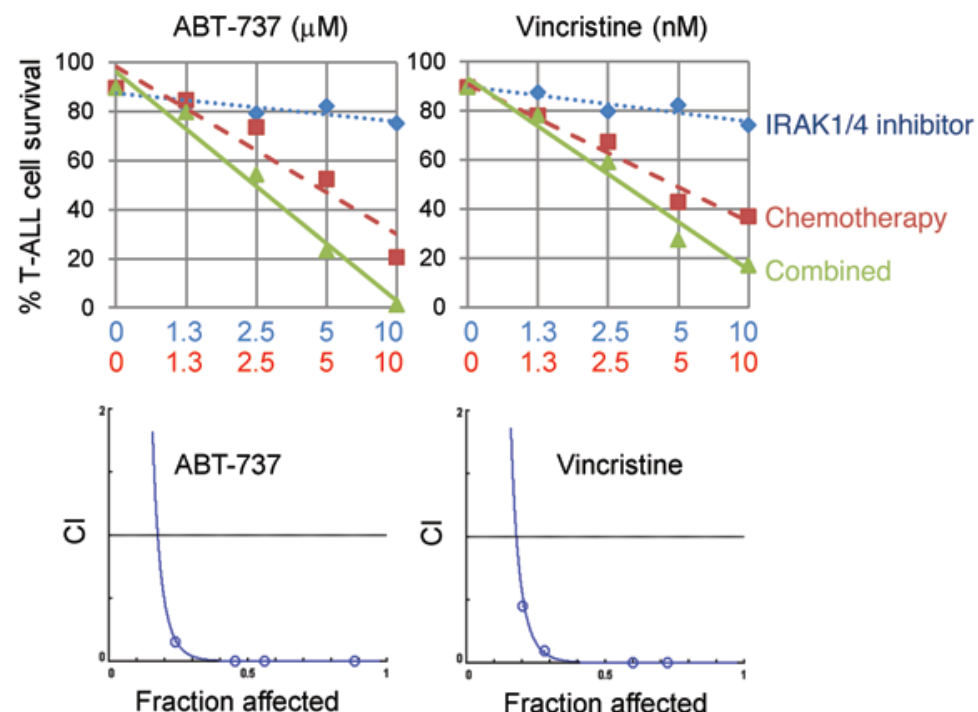
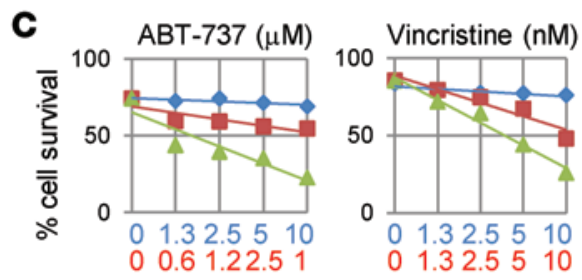

IRAK1/4 inhibitor Chemotherapy Combined

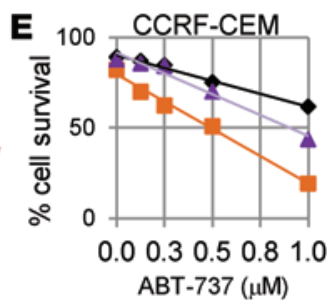

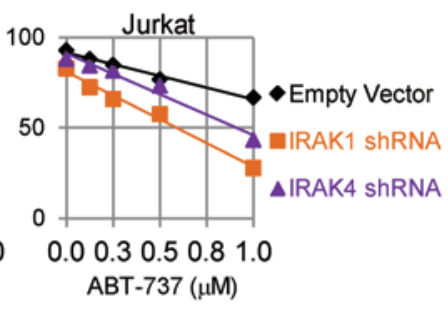

D

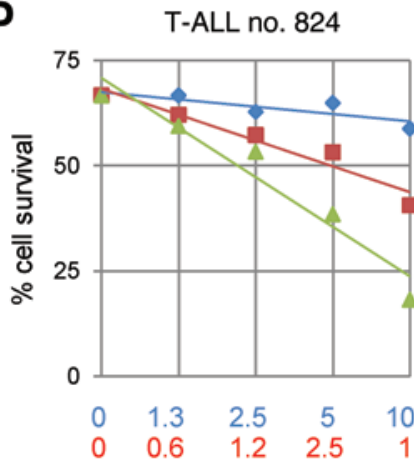

T-ALL no. 825

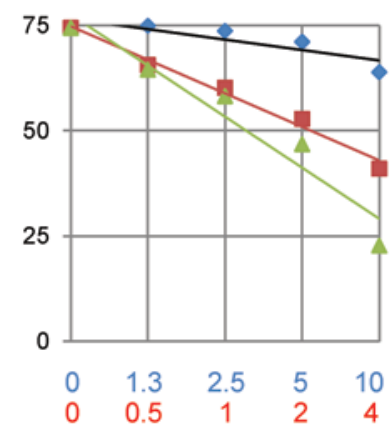

T-ALL no. 828

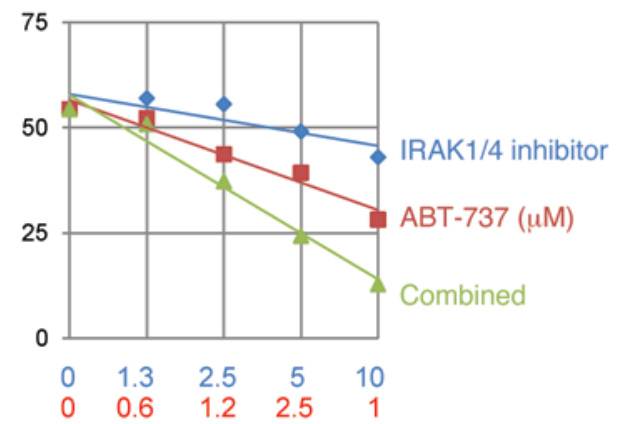

Figure 5. Identification of therapeutic compounds that synergize with IRAK1/4 inhibitor. (A) CCRF-CEM cells $\left(3 \times 10^{4}\right)$ were cultured in 96-well plates containing $2.5 \mu \mathrm{M}$ IRAK1/4 inhibitor, with or without chemotherapeutic drugs, at the indicated concentrations. After 48 hours, proliferation was determined by measuring ${ }^{3}[\mathrm{H}]$ thymidine incorporation. Heatmap based on fold changes resulting from IRAK1/4 inhibitor alone or IRAK1/4 inhibitor plus chemotherapy. (B and C) CCRF-CEM and Jurkat cells $\left(3 \times 10^{4}\right)$ or $(\mathbf{D})$ patient T-ALL cells $\left(1 \times 10^{5}\right)$ were cultured with IRAK1/4 inhibitor and/or chemotherapy at the indicated concentrations. After 48 hours, viability was determined by flow cytometry with $\mathrm{PI}$ and annexin $\mathrm{V}$ staining. Synergy between compounds is shown as the combination index (CI), evaluated using CompuSyn software. (E) CCRF-CEM and Jurkat cells were transfected with IRAK1 shRNA or IRAK4 shRNA and sorted based on GFP expression followed by treatment with ABT-737 at the indicated concentrations. Apoptosis was determined 48 hours later by flow cytometry with $\mathrm{PI}$ and annexin $\mathrm{V}$ staining. With the exception of the initial drug screen in $\mathbf{A}$, all results shown are representative of at least 3 independent experiments, each yielding similar trends.

We next examined whether the effects on p-p65, p-mTOR, p-AKT, and MCL1 expression levels also occurred in T-ALL patient samples explanted from IRAK inhibitor-treated mice. NSG mice harboring T-ALL were treated with IRAK1/4 inhibitor on days 3, 6, and 9, and T-ALL cells were collected on day 21 and the expression of various proteins examined ex vivo. As shown in Figure 7B, IRAK1/4 inhibitor treatment dramatically reduced
MCL1 expression levels. Similarly, IRAK inhibition reduced p-mTOR levels between 2.2- and 10-fold in 3 of 4 samples analyzed (Figure 7B). IRAK inhibitor treatment had moderate effects on p-p65 levels. Altogether, these data indicate that IRAK1/4 inhibition in T-ALL cells reduces leukemia progression in vivo in part through interplay with various key signaling molecules associated with cell survival and protein synthesis. 
A
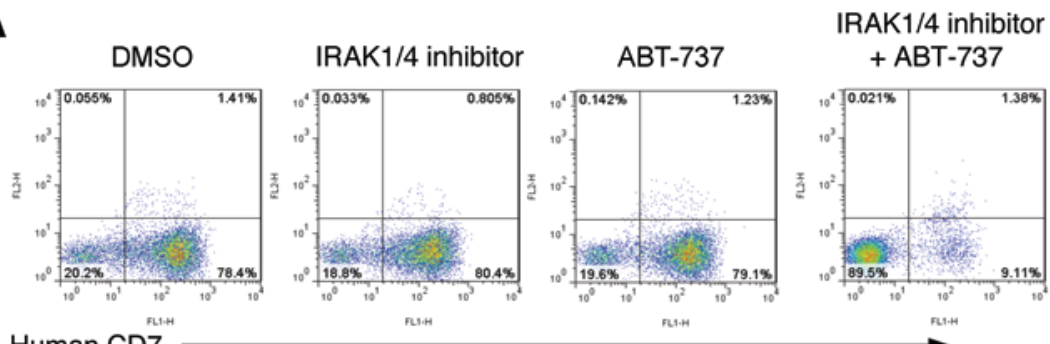

\section{B}

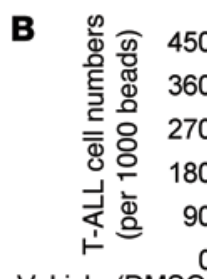

IRAK1/4 inhibitor ABT-737

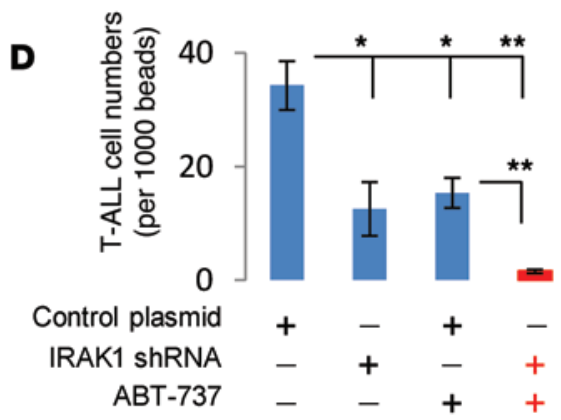

Vehicle (DMSO)

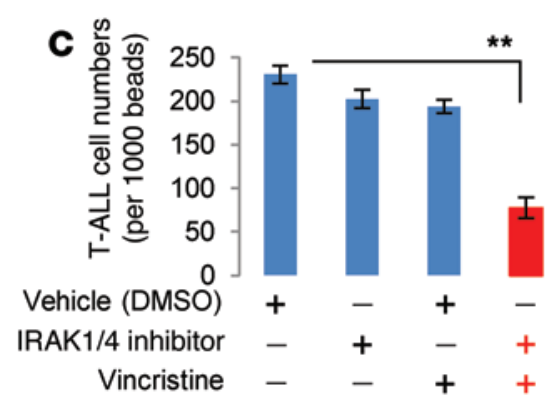

E

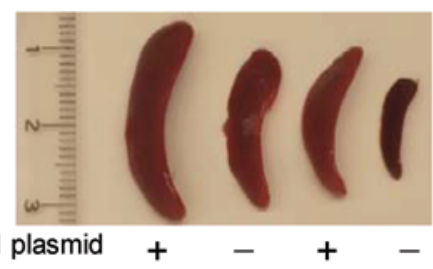

Control plasmid +-++ABT-737 - -++
$\mathbf{F}$

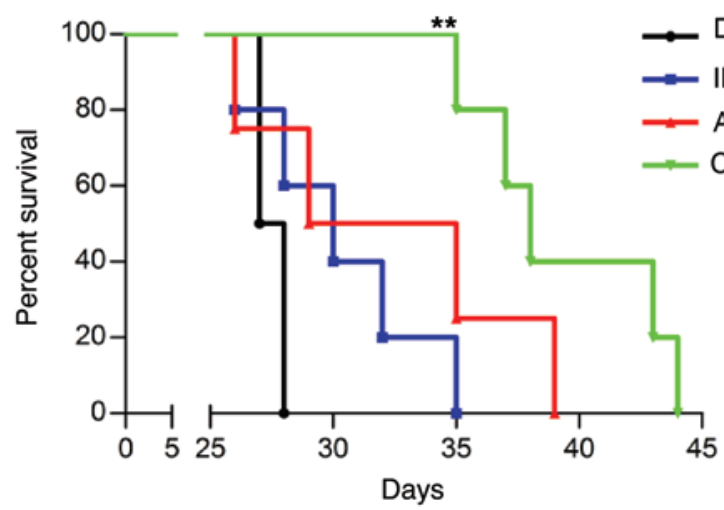

IRAK1/4 inhibitor decreases MCL1 protein stability. Both NF- $\mathrm{KB}$ and AKT signaling can regulate MCL1 expression (39-41). Because TLR stimulation has been shown to activate NF- $\kappa$ B in various cell lines and since IRAK4/1 inhibition reduced p-p65 levels, we first sought to determine whether the decrease in MCL1 levels was due to reduced transcription of Mcl1. However, IRAK inhibitor did not alter Mcl1 transcript levels (Supplemental Figure 9B), suggesting that the reduction in MCL1 levels was not due to inhibition of NF- $\mathrm{KB}$ activation. Previous studies from our group indicated that stimulating TLR1-TLR2 on primary $\mathrm{T}$ cells increased mTOR activity and resulted in increased protein biosynthesis (6). We determined whether inhibition of the MTOR pathway by IRAK inhibitor was directly associated with MCL1 protein translation by examining ribosomal fractions. We found that IRAK
Figure 6. Combination treatment with IRAK1/4 inhibitor and ABT-737 or vincristine reduces T-ALL burden and prolongs host survival. (A, B, and C) NSG mice ( $n=5 /$ group) were injected i.v. with CCRF-CEM cells $\left(3 \times 10^{6}\right)$. On days 3,6 , and 9 , mice were i.p. injected with IRAK1/4 inhibitor ( $5 \mathrm{mg} / \mathrm{kg})$, ABT-737 $(40 \mathrm{mg} / \mathrm{kg})$, or vincristine $(0.5 \mathrm{mg} / \mathrm{kg})$. T-ALL cell numbers were determined on day 21 by flow cytometry using anti-human CD7. Panel A shows representative dot plots from BM T-ALL cells. (B and C) T-ALL cell numbers from BM. (A-D) Average T-ALL cell numbers from 5 mice $( \pm \mathrm{SD}) .{ }^{*} P<0.05$ and ${ }^{* *} P<0.01$ by Student's $t$ test. (D) Jurkat cells $\left(3 \times 10^{6}\right)$ stably expressing IRAK1 shRNA or control shRNA were injected i.v. into NSG mice ( $n=5$ /group). On days 3,6 , and 9 , the mice were treated with ABT-737, and T-ALL cell numbers in BM were determined by flow cytometry. (E) Representative images of spleens collected from each of the different treatment groups. (F) NSG mice ( $n=5$ /group) were injected i.v. with CCRF-CEM cells $\left(3 \times 10^{6}\right)$ and treated with IRAK1/4 inhibitor and ABT-737 alone or together on days 3 , 6 , and 9. Survival and power values (log-rank test) are shown. ${ }^{* *} P<0.01$ versus DMSO; $P<0.01$ for combination versus IRAK1/4 inhibitor; and $P<0.05$ for combination versus ABT-737.

inhibitor moderately reduced MCL1, but not $\beta$-actin, protein biosynthesis, as demonstrated by fewer transcripts in the polyribosome fractions (Figure 7C). While altering MCL1 biosynthesis will certainly impact protein levels, MCL1 concentrations are primarily regulated by protein stability, as this protein is quickly degraded upon synthesis $(42,43)$. We examined whether inhibiting IRAK activity or, conversely, stimulating IRAK signaling with TLR1-TLR2 agonist alters the half-life of MCL1 protein. T-ALL cells were treated with IRAK1/4 inhibitor, TLR1-TLR2 ligand, or DMSO, followed by the addition of cycloheximide (CHX), to inhibit protein translation over the course of 10 hours. As shown in Figure 8A, IRAK1/4 inhibitor decreased MCL1 stability over the course of 10 hours (Figure 8A). Moreover, at earlier time points, IRAK inhibition was more effective at reducing MCL1 protein stability than was rapamycin (Supplemental Figure 10E). The synergistic effects of combinatorial therapy were more apparent when using IRAK inhibitor compared with the use of rapamycin, which might be due to the difference in kinetics between IRAK inhibitor and rapamycin (Supplemental Figure 10, F and G). In contrast, TLR1-TLR2 ligand enhanced MCL1 stability (Figure 8A). Importantly, although IRAK inhibition reduced MCL1 stability, it did not impact BCL2 or BCL-xL expression levels, highlighting a specific link between IRAK signaling and MCL1 levels.

The E3 ubiquitin ligase TRAF6 interacts with MCL1, and altering IRAK signaling changes K63-linked MCL1 ubiquitination levels. Activation of IRAK4 results in phosphorylation of IRAK1, which in turn 
A

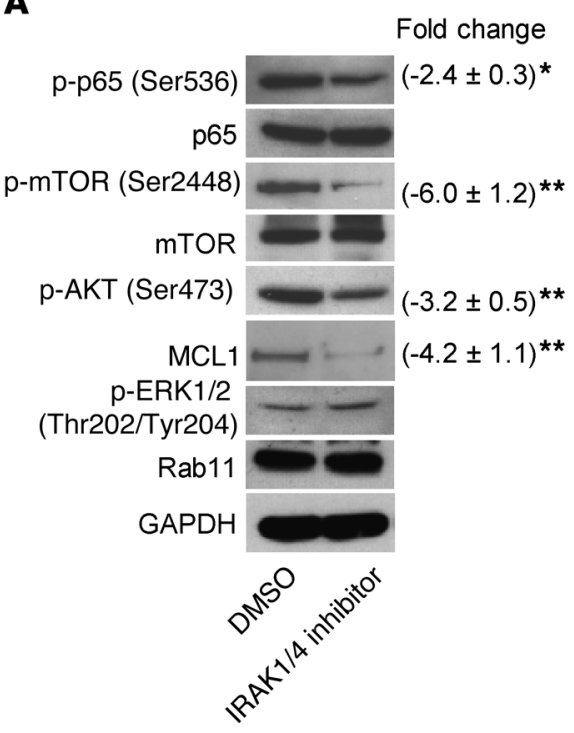

B

Patient T-ALL from

- - - - IRAK1/4 inhibitor-treated mice---

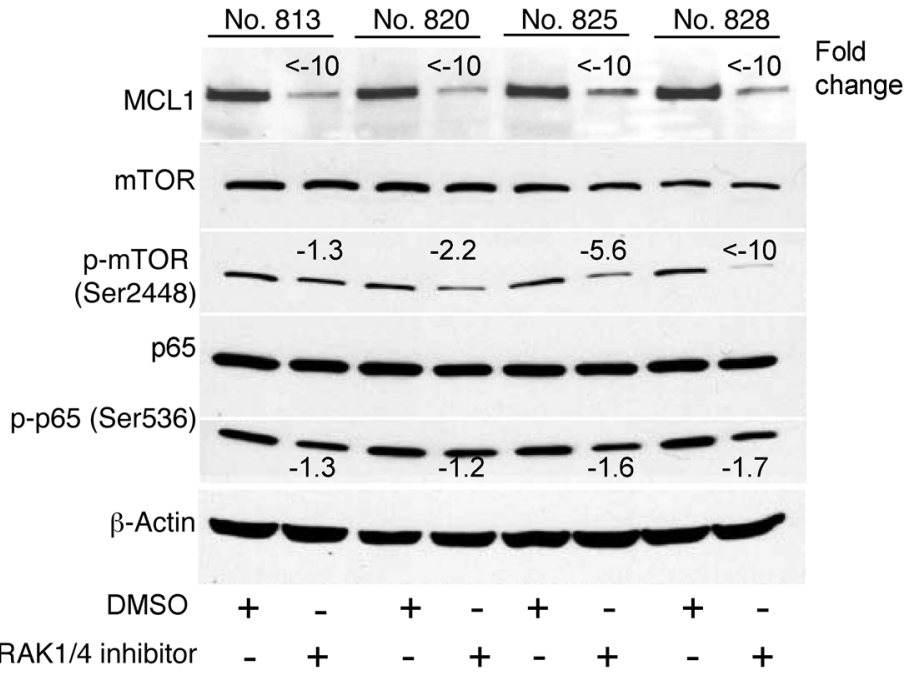

C
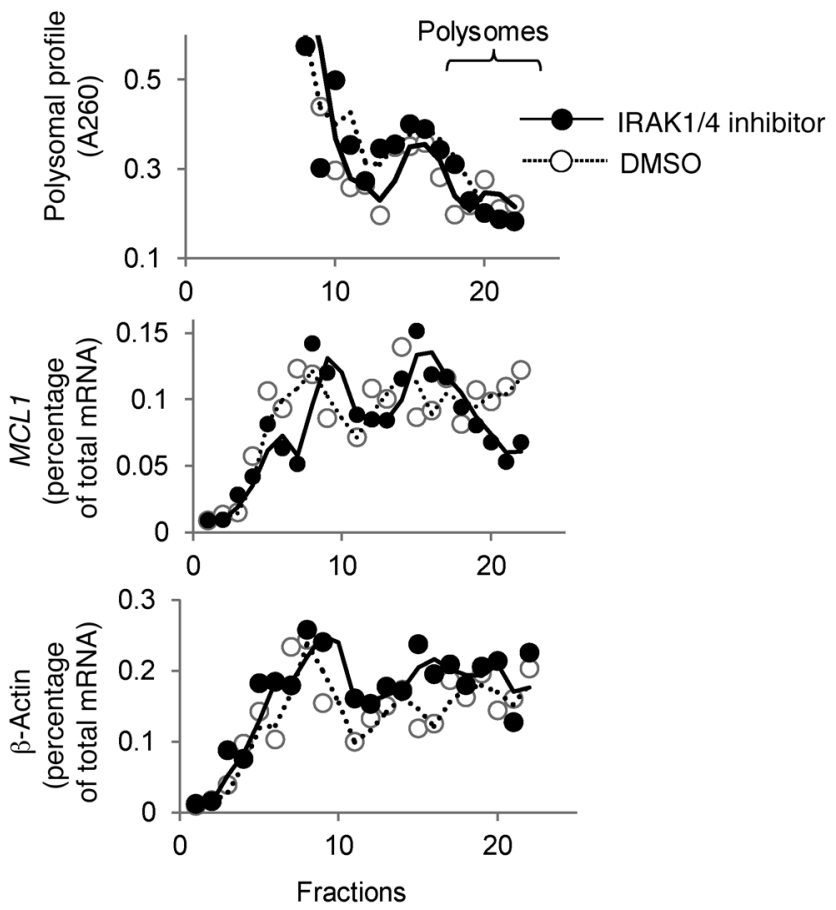

promotes association with TRAF6. TRAF6 is an E3 ubiquitin ligase whose activity has been shown to play a critical role in signaling activation and protein trafficking through K63 ubiquitination. This is in contrast to ubiquitination through $\mathrm{K} 48$, which generally targets proteins for degradation. To determine whether TRAF6 associated with MCL1, we performed co-IP assays. As shown in Figure 8B, MCL1 was detected in IPs using anti-TRAF6 antibodies. IRAK inhibition dramatically reduced MCL1 and TRAF6 association (Figure 8B). In contrast, the addition of TLR1-TLR2 ligand increased TRAF6 binding to MCL1 (Supplemental Figure 9D). The changes in association between MCL1 and TRAF6 were not due to uneven loading of precipitates, as demonstrated by equal amounts of TRAF6 and $\beta$-actin (Figure 8B and Supplemental Figure 9D). IRAK inhibition also reduced the levels of Ub-K63, whereas
Figure 7. IRAK1/4 inhibition impacts the expression levels of signaling molecules critical for T-ALL survival. (A) CCRF-CEM cells were treated with IRAK1/4 inhibitor $(5 \mu \mathrm{M})$ or DMSO for 48 hours, at which point the expression levels of the indicated proteins were determined by Western blot analysis. Average densitometric values $( \pm S D)$ of 3 independent experiments are shown ${ }^{*} P<0.05$ and ${ }^{*} P<0.01$ by Student's $t$ test. (B) NSG mice $(n=3)$ were injected with T-ALL cells from 4 different patients, followed by treatment with IRAK1/4 inhibitor ( $5 \mathrm{mg} / \mathrm{kg}$ i.p.) or vehicle control on days 3,6 , and 9. T-ALL cells were collected on day 21 , and expression levels of the indicated proteins were determined by Western blot analysis. Change in protein expression levels in DMSO and IRAK1/4 inhibitor-treated samples is shown. (C) Top panel: Representative polysomal A260 profile (of 3 independent experiments) from IRAK1/4 inhibitor- or DMSO-treated CCRF-CEM cells separated by velocity sedimentation on a $10 \%-50 \%$ sucrose gradient. Middle and bottom panels: Distribution of MCL1 and $\beta$-actin mRNA as a percentage of total mRNA in CCRF-CEM cells treated with IRAK1/4 inhibitor or DMSO control quantified by RT-PCR from the indicated fractions.
TLR1-TLR2L increased K63-linked MCL1 ubiquitination (Figure $8 \mathrm{C}$ and Supplemental Figure 9E). To validate that TRAF6 impacts MCL1 expression levels, we knocked down TRAF6 and observed that reduced TRAF6 levels correlated directly with decreased MCL1 levels in comparison with either 0-hour transfection or with cells transfected with a control plasmid (Figure 8D). In contrast, we found that knocking down TRAF6 did not impact BCL2 or BCL-xL, further indicating a preferential link between TRAF6 and MCL1. These results indicate that TRAF6 associates with MCL1 and suggest that this leads to the K63 ubiquitination of MCL1, thereby prolonging elevated MCL1 protein levels in T-ALL cells.

Overexpressing MCL1, but not BCL-xL, rescues $T-A L L$ cells from IRAK1/4 inhibitor-ABT-737-induced apoptosis in vitro. We sought further evidence that MCL1 plays a key role in the ability 
A

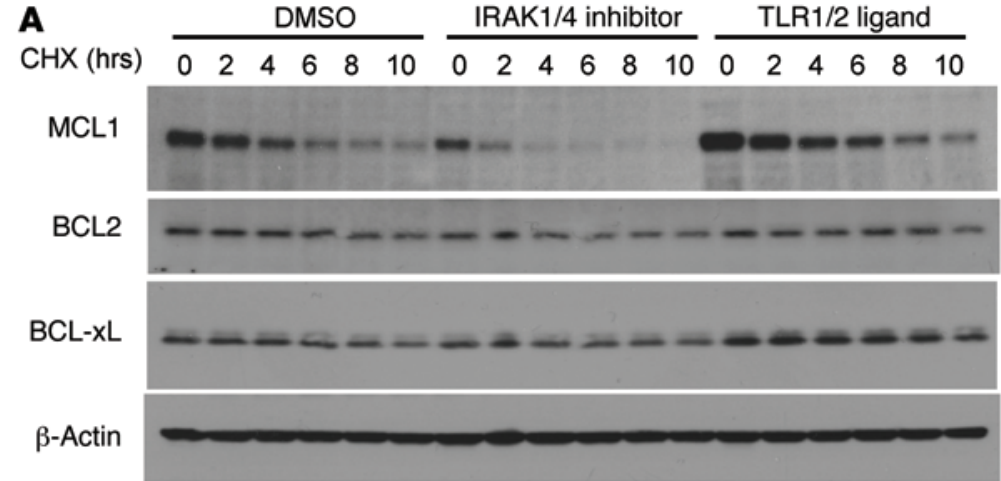

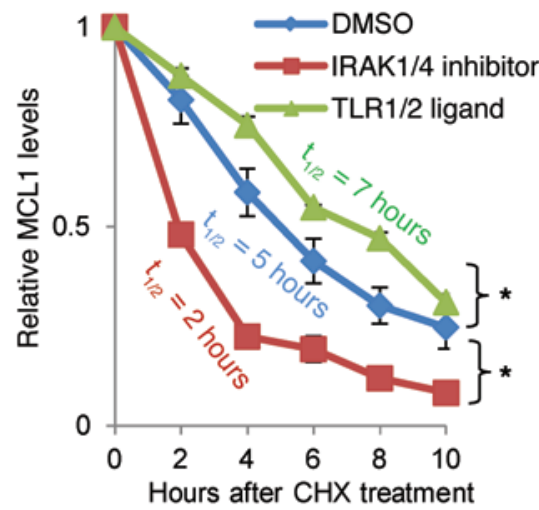

Hours after $\mathrm{CHX}$ treatment
B

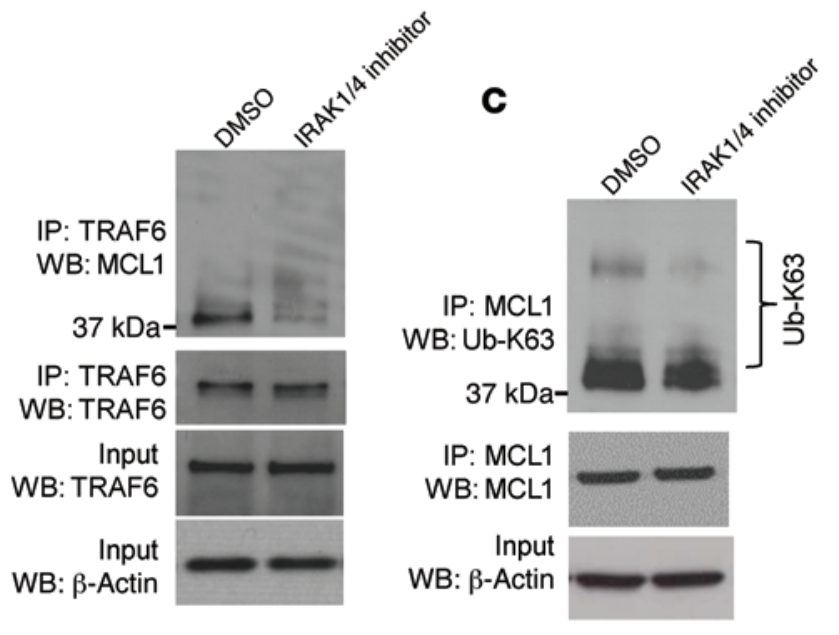

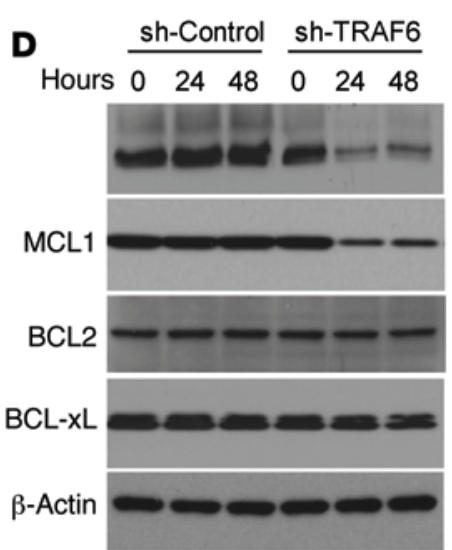

$\mathbf{E}$

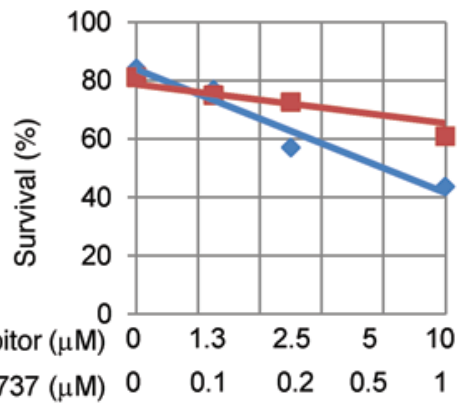

- p-Vector $\square$ p-MCL1

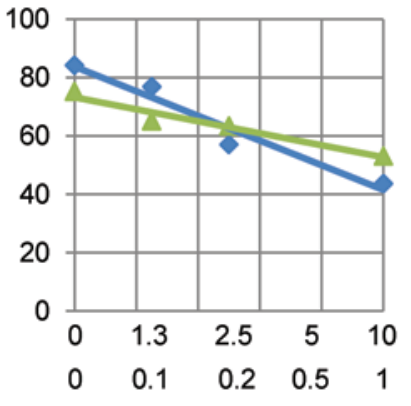

- p-Vector $\triangle \mathrm{p}$-IKK2

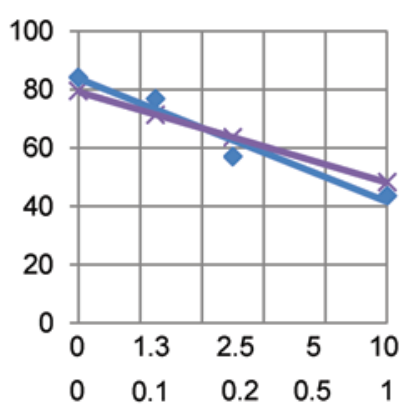

- p-Vector $\times$ p-myrAKT

Figure 8. IRAK4/1 signaling impacts MCL1 protein stability, and MCL1 interacts with TRAF6. (A) CCRF-CEM cells were treated with IRAK1/4 inhibitor $(5 \mu \mathrm{M})$ or DMSO for 6 hours. Cells were then treated with CHX for $0,2,4,6,8$, and 10 hours and cell lysates analyzed by Western blotting for MCL1, BCL2, and BCL-XL expression. Densitometric values for MCL1 protein relative to the 0 -hour time point for each treatment group are summarized in the graph. Mean densitometric values ( \pm SD) from 3 independent experiments are shown. ${ }^{*} P<0.05$ by Student's $t$ test. (B and C) Co-IP assay and Western blot analysis. CCRF-CEM cells were treated with IRAK1/4 inhibitor $(5 \mu \mathrm{M})$ or DMSO, and lysates were subjected to co-IP using anti-TRAF6 antibody (B) or antiMCL1 antibody (C) and immunoblotted with the indicated antibodies. (D) CCRF-CEM cells were transfected with TRAF6 knockdown (sh-TRAF6) or control (sh-Control) plasmids, and expression levels of the indicated proteins were analyzed by Western blotting. (E) CCRF-CEM cells were transfected with control plasmid ( $p$-Vector) and plasmids to overexpress MCL1 ( $p$-MCL1), mutated IKK2 (p-IKK2), and AKT ( $p$-myrAKT) and then treated with the indicated drugs at various concentrations. Apoptosis $\left(\mathrm{Pl}^{+}\right.$annexin $\mathrm{V}^{+}$) was measured by flow cytometry 48 hours after treatment. Results shown are representative of 3 (A and $\mathbf{E})$ or 2 (B-D) independent experiments.

of IRAK1/4 inhibitor to augment the cytotoxic effects of ABT-737. Both CCRF-CEM and Jurkat T-ALL cells overexpressing MCL1 were partially rescued from the cytotoxic effects of IRAK1/4 inhibitor and ABT-737 combination treatment; these effects were more pronounced at the higher drug concentrations (Figure 8E and Figure 9A); we confirmed the synergy of both drugs by Chou-Talalay analyses. Furthermore, because inhibition of IRAK1/4 signaling also reduced the activation levels of NF- $\mathrm{kB}$ and AKT, we tested whether overexpressing mutated IKK2, which results in NF- $\mathrm{KB}$ (p65) activation, or overexpressing myristoylated AKT, which results in constitutive activation of AKT and phosphorylation of mTOR, would also rescue cells from combination therapy $(44,45)$. However, unlike overexpression of MCL1, we found that restoring these signaling pathways through overexpression did not rescue 

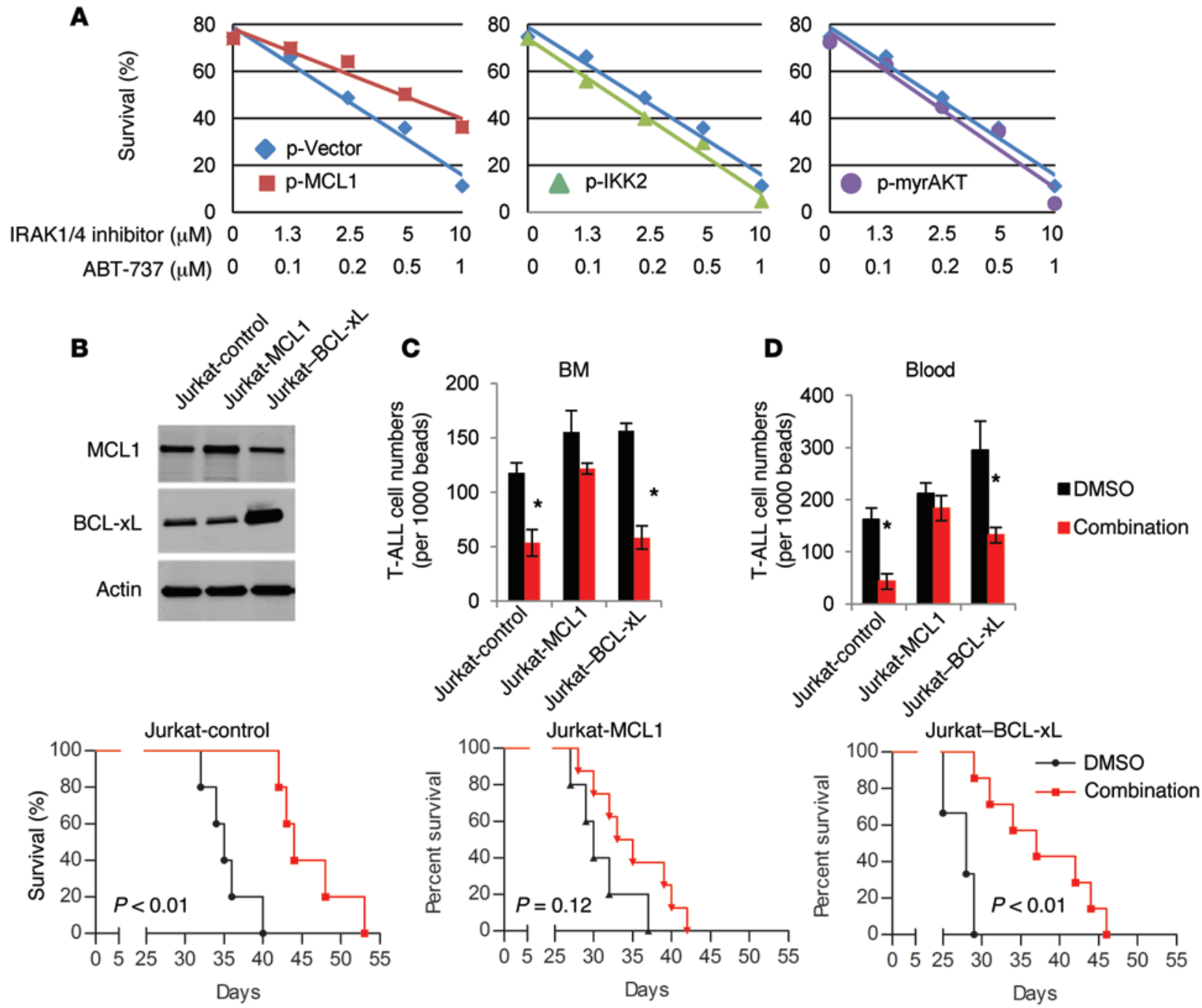

Figure 9. MCL1, but not BCL-xL, overrides the therapeutic effects of combinatorial IRAK1/4 inhibitor and ABT-737 therapy in vivo. (A) Jurkat cells were transfected with control plasmid ( $p$-Vector) and plasmids to overexpress MCL1 ( $p-M C L 1)$, mutated IKK2 ( $p-I K K 2)$, and AKT ( $p$-myrAKT) and then treated with the indicated drugs at various concentrations. Apoptosis ( $\mathrm{Pl}^{+}$annexin $\mathrm{V}^{+}$) was measured by flow cytometry 48 hours after treatment. (B) Western blot analysis of MCL1 and BCL-xL expression in stably transduced Jurkat-MCL1, Jurkat-BCL-xL, or Jurkat control cells. Data in $\mathbf{A}$ and $\mathbf{B}$ are representative of 3 independent experiments. (C and D) NSG mice were injected i.v. with MCL1- or BCL-xL-overexpressing Jurkat cells $\left(2 \times 10^{6}\right)$ or control Jurkat cells. Mice were injected i.p. with IRAK1/4 inhibitor $(5 \mathrm{mg} / \mathrm{kg})$ and ABT-737 $(40 \mathrm{mg} / \mathrm{kg})$ or vehicle control on days 3, 6, and 9, and the number of CD5 ${ }^{+}$T-ALL cells, shown as the average number of CD5+ T-ALL cells in BM and peripheral blood from 5 mice, was measured by flow cytometry on day $21 .{ }^{*} P<0.05$ by Student's $t$ test. (E) Survival of NSG mice injected i.v. with the indicated cell lines $\left(2 \times 10^{6} /\right.$ mouse $)$ and treated with IRAK1/4 inhibitor plus ABT-737 on days 3 , 6 , and 9. Data represent a compilation of 2 independent experiments, with 4 or 5 mice per group. $P$ values were determined using the log-rank test.

CCRF-CEM T-ALL cells from the cytotoxic effects of ABT-737 plus IRAK inhibition (Figure 8E). That overexpression of these vectors resulted in increased expression of the intended proteins was verified by Western blot analysis (Supplemental Figure 9C). Together, these data indicate that the chemo-enhancing effects of IRAK1/4 are primarily mediated through its ability to regulate MCL1 protein levels (although alterations in other signaling pathways such as mTOR may also potentiate this process).

Overexpressing MCL1, but not $B C L-x L$, reverses the therapeutic benefits of IRAK1/4 inhibitor and ABT-737 combination treatment in vivo. To further validate the role of MCL1 in response to combinatorial IRAK inhibitor plus ABT-737 treatment, we examined the progression of Jurkat T-ALL cell lines engineered to stably overexpress MCL1 or BCL-xL (Figure 9B). As shown in Figure 9, C and D, control
T-ALL and BCL-xL-overexpressing T-ALL cells responded to combination treatment and demonstrated reduced numbers of T-ALL cells in the BM and blood as compared with the numbers detected in DMSO-treated control mice. In sharp contrast, we found that mice with T-ALL cells overexpressing MCL1 did not respond to combination treatment and that they had T-ALL cell numbers equal to those detected in control mice (Figure 9, C and D). Furthermore, while combination therapy prolonged the survival of mice with T-ALL or BCL-xL-overexpressing T-ALL cells as compared with survival of control mice, the therapeutic effect was lost in mice with MCL1-overexpressing T-ALL cells (Figure 9E). Collectively, these data demonstrate that overexpression of MCL1 overcomes the beneficial effects of combination therapy and further underscore the important interplay between IRAK signaling and MCL1. 


\section{Discussion}

The proliferative and prosurvival effects of stimulating MyD88/ IRAK signaling in primary CD4 and CD8 $\mathrm{T}$ cells prompted us to examine the potential biological significance of this signaling pathway in T cell neoplasms. Our studies are the first to our knowledge to examine in detail the prosurvival-, proliferation-, and chemoresistance-conferring effects of IRAK1/4 signaling in T-ALL. A major finding of this study was that T-ALL cells express elevated levels of p-IRAK1 and p-IRAK4 and that inhibition of this signaling pathway using a small-molecule inhibitor or shRNA dramatically reduced T-ALL proliferation. Perhaps more important, however, was that IRAK inhibition augmented the cytotoxic activity of various types of antileukemic drugs. These studies are also the first to our knowledge to characterize the proliferative effects of various TLR agonists on $\mathrm{T}$ cell neoplasms. Collectively, these studies indicate that IRAK1/4 signaling in T-ALL cells play a critical role in their proliferation and survival and identify IRAK4/IRAK1 as a therapeutic target to prevent or overcome chemoresistance.

Understanding the molecular signals that impart an ability of T-ALL cells to overcome the cytotoxic effects of chemotherapy is a critical step in developing more effective therapies. Given that IRAK inhibition drastically reduced proliferation but had only a moderate effect on cell viability, we screened nearly 500 therapeutic compounds and identified 22 compounds that were effective at killing T-ALL cells in vitro when combined with IRAK1/4 inhibitor. The majority of the drugs identified could be categorized into 4 targeted pathways, in the order of most to least effective: BCL inhibitors (ABT-737), agents that disrupt microtubules (vincristine), and, to a lesser extent, HSP90 and EGFR inhibitors. We validated the antileukemic effects of combined therapy in mice with established T-ALL and investigated the mechanisms through which IRAK inhibition regulates chemoresistance of T-ALL cells by examining the expression levels of nearly 250 cell survivalrelated proteins. In particular, we found significant reductions in the levels of MCL1, p-mTOR, p-AKT, and p-p65. IRAK inhibition had little effect on BCL-xL and BCL2 basal levels. Overexpression of MCL1 rescued cells from the cytotoxic effects of IRAK1/4 inhibitor combination therapy, but overexpression of p-p65, AKT, or BCL-xL failed to compensate for the loss of IRAK signaling (Figure 8 and Figure 9). It is important to note that although MCL1 is a BCL family member known for its role in cell survival, it also appears to play an indirect, but very important, role in proliferation (46). We found that knocking down MCL1 in T-ALL moderately reduced proliferation, while having minimal effects on apoptosis (Supplemental Figure 10, A-D). MCL1 interacts with BAX and BAK and prevents their activation. Bergamo reported that ABT-737 was ineffective at downregulating prosurvival BCL2 and BCL-xL signaling and that treatment with ABT-737 resulted in a compensatory increase in MCL1 expression in AML cells (47). We postulate that the synergy between ABT-737 and IRAK inhibition is due to disruption of BCL2 and BCL-xL by ABT-737, combined with dramatic reductions in MCL1 levels by inhibition of IRAK. Reductions in p-mTOR and NF- $\kappa B$ levels in response to IRAK inhibition also likely contributed to the observed synergistic effects.

Various genetic alterations are known to give rise to T-ALL, including activating mutations in Notch 1 (48), aberrant expression of LMO1 and LMO2, and deletions of the CDKN2A locus, which are prevalent in T-ALL cases $(49,50)$. Two recent reports described a somatic mutation in MyD88 (L265P) in Waldenström macroglobulinemia and in diffuse large $B$ cell lymphoma (DLBCL) that promotes malignant progression in part by constitutive NF- $\mathrm{B}$ activity $(29,30)$. Considering the importance of the MyD88 L265P mutation, its role in activating IRAK signaling, and the fact that IRAK1 and IRAK4 are constitutively activated in CCRF-CEM and Jurkat T-ALL cell lines, we sequenced the MYD 88 gene in these 2 cell lines, but found them to contain the WT MyD88 (Supplemental Figure 3B). Moreover, it appeared that IRAK inhibition impaired T-ALL proliferation and sensitized cells to ABT-737 or vincristine, regardless of the genetic abnormalities typically associated with T-ALL, and no clear pattern emerged, due in part to our limited sample number (Supplemental Table 2). These data do not rule out the possibility that some T-ALL samples might express activating mutations in MYD88 (or in other IRAK4 signaling-related genes or other related genes), but indicate that IRAK signaling might occur by other mechanisms. The prevalence of MYD 88 mutations in T cell neoplasms has yet to be clearly defined, but is an active part of our research endeavors; nevertheless, our data suggest that IRAK4 signaling might represent a more generalized therapeutic target applicable to T-ALL cases with different types of genetic alterations.

Can MyD88/IRAK signaling in T cell neoplasms contribute to disease progression in patients? A role for MyD88/IRAK signaling in $\mathrm{T}$ cell neoplasms has not been thoroughly examined, but a possible role of this pathway in malignant $\mathrm{T}$ cell progression was described in HTLV-infected T cells. Mizobe demonstrated that MyD88 was constitutively associated with IRAK1 in HTLV-Itransformed $\mathrm{T}$ cells, resulting in constitutive activation of $\mathrm{NF}-\kappa \mathrm{B}$. MyD88-IRAK1 association resulted in increased levels of IL-6 as well as enhanced proliferation and survival (51). Our studies demonstrate that TLR stimulation induced T-ALL proliferation in vitro and in tumor-bearing mice (Figure 3) and that treatment with IRAK1/4 inhibitor reduced T-ALL expansion in tissue culture and in mice with T-ALL (Figure 4, Figure 6, and Figure 9). These data raise the possibility that TLR agonists (or other pattern recognition receptor agonists) might contribute to T-ALL proliferation and perhaps chemoresistance in patients. The effects of TLR ligands might have occurred indirectly by activating TLR/IRAK signaling on endogenous mouse cells. However, a T-ALL sample that did not respond to TLR2 ligand in vitro was also unresponsive to TLR2 ligand in vivo, suggesting that the proliferative effects of TLR ligand were due to direct TLR2 engagement on T-ALL cells in vivo (Supplemental Figure 4E). These data raise the question of the source of TLR ligands in vivo, which is a significant issue in the biology and pathogenesis of T cell malignancies. TLRs can recognize a wide range of endogenous danger-associated molecular patterns (DAMPs), or so-called alarmins (52), released from dying or stressed cells. Among these alarmins are members of the heatshock protein (HSP) family, which have been shown to activate TLR2 and TLR4 (53-56). Endogenous TLR9 and TLR2 agonists released by damaged tissue can also stimulate TLRs on T cells (57, 58). TLR expression in $\mathrm{T}$ cell malignancies has also been shown by Smith (59) and Jarrousse (60), who found TLR1, TLR2, TLR4, and TLR9 to be highly expressed in peripheral $\mathrm{T}$ cell lymphoma and Sézary syndrome cutaneous lesions. Despite the inability of some T-ALL cells to respond to TLR2 ligand, the importance of 
IRAK signaling in T-ALL progression in vivo was supported by our findings that knocking down IRAK1 in T-ALL cells reduced growth in vivo (Figure 6D) and that T-ALL cells collected from IRAK inhibitor-treated mice exhibited dramatic decreases in MCL1 and p-mTOR levels (Figure 7). Collectively, these data highlight the biological effects that activating IRAK signaling have on T-ALL progression, strengthening the possibility of using IRAK inhibitors as a targeted therapy.

Our studies describe for the first time to our knowledge how dysregulated and constitutively active IRAK1/4 signaling in T-ALL results in enhanced MCL1 protein stability. We further demonstrate that the activation of TRAF6 leads to its association with MCL1 and increased K63 ubiquitination. In our model (depicted in Supplemental Figure 11), the reduction in MCL1 that occurs with IRAK1/4 inhibitor is associated with chemotherapy sensitization. TRAF6 has been shown to ubiquitinate other substrates that might play an active role in cancer progression (61-63). Liu reported that TRAF6 signaling in multiple myeloma (MM) resulted in NF- $\kappa B$ and JNK activation and played a critical role in MM proliferation (61), while Linares et al. showed that TRAF6 is necessary for translocation of mTORC1 to lysosomes and that TRAF6 catalyzed K63 ubiquitination through its ability to interact with the signaling adaptor p62 raptor, a component of the mTORC1 pathway (15). Yang's observation that TRAF6 was essential for AKT ubiquitination and phosphorylation was in alignment with our observation that IRAK4/1 inhibition reduced p-AKT levels (16).

In summary, recent advances underscore the biological importance of TLR/MyD88/IRAK signaling in primary T cells, and our studies suggest that this signaling pathway plays an important role in the pathogenesis and progression of T-ALL and perhaps other $\mathrm{T}$ cell malignancies. IRAK inhibition slows the growth of T-ALL, and, perhaps more important, blocking IRAK4/1 signaling enhances the effects of chemotherapeutic drugs. These studies indicate that interfering with IRAK4 signaling in T-ALL might serve as a novel therapeutic strategy to enhance the efficacy of chemotherapeutic drugs.

\section{Methods}

Human T cell leukemia cell lines, patient samples, and culture medium The 293T/17, CCRF-CEM, CEM/C1, CEM/C2, CEM-C7, Jurkat, Loucy, MOLT-4, HSB-2, SUP-T1, Hut 78, HH, Hut 102, MO, and H9 T leukemia and Tymphoma cell lines were purchased from the ATCC during the 2 years prior to submission of this manuscript. Breast cancer cell lines MDA-MB-231, MDA-MB-468, SBR3, and MCF-7, were provided by Xinrong Ma (University of Maryland School of Medicine) and RAJI, JEKO, RAMOS, and SP50 by Tonya Webb (University of Maryland School of Medicine). All of the cell lines were initially expanded and cryopreserved within 1 month of their receipt. Cells were typically used for 6 months, at which time a fresh vial of cryopreserved cells was used. Total $\mathrm{T}$ cells from healthy donors were isolated via negative selection followed by positive selection using a $\mathrm{CD}^{+}$selection kit (Invitrogen). T cells were isolated from T-ALL patients' BM or peripheral blood samples containing more than $70 \%$ leukemia cells using a CD2 ${ }^{+}$isolation kit (STEMCELL Technologies). For in vitro experiments, T-ALL cells were cultured in AIM-V medium supplemented with $20 \%$ human $A B$ serum in the presence of recombinant human stem cell factor ( $50 \mathrm{ng} / \mathrm{ml}$; Amgen), insulin (20 nM; Sigma-Aldrich), and IL-7 (50 ng/ml; R\&D Systems). In other experiments, protein lysate was collected using the Full Moon BioSystems buffer prior to protein examination by Western blot analysis or protein array.

\section{Microarray data sets and data analysis}

All of the microarray data sets were from publicly available microarray data resources. Two of them were from Gene Expression Omnibus (GEO GSE36133 and GSE46170). Sabina Chiaretti of the University of Rome "La Sapienza" (Rome, Italy) provided microarray data sets from Chiaretti et al. (38) and Yeoh et al. (64) for analysis of IRAK1 mRNA levels in patients with ongoing remission or relapse. Data analysis was performed using the BRB-ArrayTool developed at the Biometric Research Branch, Division of Cancer Treatment and Diagnosis of the National Cancer Institute (NCI). IRAK4 mRNA levels in 917 cancer cell lines were from Oncomine (https://www.oncomine.com).

\section{Real-time PCR}

Real-time PCR (RT-PCR) and quantitative PCR (qPCR) array assays were previously described (6). The primers used for qPCR are listed in Supplemental Table 3.

\section{Antibodies}

Western blot analysis. The following antibodies were used: TLR1 (B-23) (sc-130896) and total IRAK1 (H-273) (sc-7883) (from Santa Cruz Biotechnology Inc.); TLR2 (12276), TLR4 (2246), TLR7 (5632), TLR9 (5845), TRIF (4596), IRAK-M (4369), IRAK2 (4367), IRAK4 (4363), TRAF6 (8028), Tollip (4748), p65 (4764), p-p65 (3033), p-mTOR (2971), mTOR (2983), AKT (9272), p-AKT (9271), p-ERK1/2 (9101), RAB11 (5589), MCL1 (4572), GAPDH (14C10) (2118), BCL2 (2870), BCL-xL (2764), p-IRAK1 (Thr209) (12756), and p-IRAK4 (Thr345/ Ser346) (11927) (from Cell Signaling Technology); TLR3 (DDX0472B) and TLR5 (IMG663C) (from Imgenex); and TLR6 (3D10H11), TLR8 (44C143), and MyD88 (from Abcam).

IF. IRAK1 (4259) and IRAK4 (4363), from Cell Signaling Technology, were used for IF staining.

Neutralizing antibody. TLR2 (PAb-hTLR2) neutralizing antibody was obtained from InvivoGen.

FACS. Human CD2 (hCD2) (561759), hCD5 (561896), hCD7 (555361), hCD45 (340942), mouse CD3 (mCD3) (561799), mCD4 (557307), mCD8 (553035), mCD11c (553802), mCD19 (557398), mNK1.1 (557391), mSca1 (562059), mc-Kit (553356), and mF4/80 (563899) were used for FACS and purchased from BD Biosciences - Pharmingen.

\section{IF staining}

CCRF-CEM cells or Jurkat T cells $\left(5 \times 10^{4}\right)$ were loaded on slides by cytospinning. After fixation and permeabilization, the cells were stained with rabbit anti-IRAK1 or -IRAK4 antibodies (Cell Signaling Technology) and were subsequently stained with fluorescence-labeled anti-rabbit secondary antibodies. Pictures were taken using an Olympus IX81 confocal microscope and Fluoroview 1000 software.

\section{TLR agonists, IRAK inhibitor, and other inhibitors}

The following TLR ligands were purchased from InvivoGen: tripalmitoyl-S-(bis(palmitoyloxy)propyl)-Cys-Ser-(Lys)3-Lys 
(TLR1/2); poly I:C (TLR3); ultrapure Salmonella minnesota LPS (TLR4); Salmonella typhimurium flagellin (TLR5); FSL1 (TLR2/6); imiquimod (TLR7); ssRNA40/lyovec (TLR8); and CpG-ODN 2006 (TLR9). In some experiments, we used the IRAK1/4 inhibitor, which is a cell-permeable benzimidazole compound that selectively inhibits IRAK1 and IRAK4 and shows little to no activity against a panel of 27 other kinases (EMD Millipore). Rapamycin was purchased from Sigma-Aldrich.

\section{Western blot and protein array analyses}

Total cell extracts were prepared from neoplastic $\mathrm{T}$ cell lines or from cells from T-ALL patients. Proteins $(20 \mu \mathrm{g} / \mathrm{lane}$ from cell lines and $\sim 60 \mu \mathrm{g} /$ lane from T-ALL patients) were resolved in Tris-glycine SDS gels and transferred to PVDF membranes. The membrane was blocked for 4 hours with $5 \%$ milk in PBS and $0.05 \%$ Tween-20, followed by incubation with antibodies overnight at $4^{\circ} \mathrm{C}$ and subsequently with HRP-conjugated secondary antibody and detection using ECL (Amersham Pharmacia Biotech). We also used a protein array from Full Moon Biosystems. For these experiments, purified T-ALL cells were incubated for 48 hours in the presence of IRAK1/4 inhibitor $(5 \mu \mathrm{M})$ or an equal volume of vehicle (DMSO). Total protein was then extracted, and the levels of various proteins were measured according to the manufacturer's protocol.

\section{IP}

Cells were lysed with ice-cold lysis buffer (20 mM Tris ( $\mathrm{pH} 7.5)$, $150 \mathrm{mM} \mathrm{NaCl}, 1 \mathrm{mM}$ EDTA, 1 mM EGTA, 1\% Triton X-100, $2.5 \mathrm{mM}$ sodium pyrophosphate, $1 \mathrm{mM} \beta$-glycerophosphate, $1 \mathrm{mM} \mathrm{Na} \mathrm{VO}_{4}$, and $1 \mu \mathrm{g} / \mathrm{ml}$ leupeptin) containing $1 \mathrm{mM}$ PMSF and 1:100 protease cocktail inhibitors. Nuclear pellets and debris were removed by centrifugation at $17 \times 10^{3} \mathrm{~g}$ for 20 minutes at $4^{\circ} \mathrm{C}$. An equivalent amount of proteins from cell lysates was incubated overnight at $4^{\circ} \mathrm{C}$ with the specified antibodies. Protein $\mathrm{A}$ beads were added to the immune complexes for 45 minutes, then washed 5 times with ice-cold lysis buffer. Purified IPs, immobilized on protein A beads, were mixed with an equal volume of $2 \mathrm{x}$ Laemmli sample buffer and boiled for 5 minutes. Proteins were resolved on SDS-PAGE for Western blot analysis.

\section{Polysome assay}

CCRF-CEM cells were treated with IRAK1/4 inhibitor or DMSO for 48 hours and then lysed in buffer containing $100 \mu \mathrm{g} / \mathrm{ml}$ cycloheximide. After removal of nuclei and mitochondria, supernatants were layered onto $10 \%$ to $50 \%$ sucrose gradients and spun at 38,000 rpm (2 hours at $4^{\circ} \mathrm{C}$ ) in an SW 40 rotor (Beckman Coulter). Centrifuged gradients were fractionated into twelve 1-ml fractions, and the polysome profile was determined via UV absorbance at $260 \mathrm{~nm}$, followed by RNA extraction, reverse transcription, and quantification by RT-PCR.

\section{Plasmids, transfection, lentiviral production, transduction, and stable cell selection}

Plasmids for TRAF6, IRAK1, and IRAK4 knockdown were obtained from InvivoGen. p-MCL1, p-IKK, and p-myrAKT were purchased from Addgene. The lentiviral constructs for MCL1 and BCL-xL overexpression and the lentiviral shRNA constructs targeting IRAK1 were purchased from Thermo Scientific. One of the 6 constructs that had the best knockdown efficiency was used in the experiments. Lentiviruses were pseudotyped with VSV-G, produced by $293 \mathrm{~T} / 17$ cells, and concentrated by ultracentrifugation at 20,000 rpm for 2 hours at $4^{\circ} \mathrm{C}$.
Cells at $1 \times 10^{5} / \mathrm{ml}$ were transduced with lentivirus at a MOI of 0.5 to approximately 1 in the presence of $8 \mu \mathrm{g} / \mathrm{ml}$ polybrene (Santa Cruz Biotechnology Inc.). Forty-eight hours after transduction, GFPpositive cells were isolated by FACS.

\section{Proliferation, cell number, and apoptosis assays}

Cells were cultured in a 96-well plate (Corning Costar) with TLR ligands $1-9$ at various concentrations, starting at $10 \mu \mathrm{g} / \mathrm{ml}$, in a final volume of $200 \mu$ l. TLR ligands underwent 2 -fold dilution. Cell lines $\left(25 \times 10^{3} /\right.$ well to $40 \times 10^{3} /$ well $)$ were cultured for 54 hours, whereas fresh patient-derived T-ALL cells $\left(200 \times 10^{3} /\right.$ well $)$ were cultured for 30 hours, prior to adding $\left[{ }^{3} \mathrm{H}\right]$-thymidine for 18 hours. After this time point, cells were harvested with a Packard Filtermate 96-well harvester (PerkinElmer) and counted using a Packard TopCount NXT scintillation and luminescence counter (PerkinElmer). Results were expressed as average cpm. A CellTiter 96 AQueous Non-Radioactive Cell Proliferation Assay kit (Promega) was used for the proliferation assay described in Figure 2A. Apoptosis was measured by flow cytometry after staining cells with annexin $\mathrm{V}$ and 7-AAD (BD Biosciences - Pharmingen). Cells were counted under microscopy after staining with trypan blue.

\section{Cell-cycle analysis}

Control and treated cells were collected, fixed, and stained with propidium iodide $(100 \mu \mathrm{g} / \mathrm{ml})$ in PBS containing sodium citrate $(1 \mathrm{mg} / \mathrm{ml})$, Triton X-100 (0.1\%), and RNAse $(20 \mu \mathrm{g} / \mathrm{ml})$ for $30 \mathrm{~min}-$ utes. Data were acquired on a BD FACScan flow cytometer using CellQuest software (BD Biosciences - Immunocytometry Systems) and analyzed with FlowJo software.

\section{High-throughput drug screening and data analysis}

Four hundred eighty-four small-molecule inhibitors, including most of the FDA-approved inhibitors, active pharmaceutical ingredients, and chemotherapeutic agents, were purchased from Selleck Chemicals (L1100). CCRF-CEM cells $\left(3 \times 10^{4}\right)$ were cultured in 96-well plates containing $2.5 \mu \mathrm{M}$ IRAK1/4 inhibitor and/or small-molecule inhibitors at 4 different concentrations $(0.015,0.060,0.250$, and $1.000 \mu \mathrm{M}$ ). Forty-eight hours later, cellular activity was measured by a tritiated thymidine incorporation assay. The raw proliferative values were then $\log _{2}$ transformed and normalized to untreated and then IRAK1/4 inhibitor-treated controls. A heatmap was generated based on the fold reduction between combined IRAK1/4 inhibitor and chemotherapy and IRAK1/4 inhibitor alone. The small-molecule inhibitors with more than a 2 -fold reduction at at least 2 concentrations were selected and further validated by synergy assay.

\section{Mice}

NSG mice (The Jackson Laboratory) and C57BL/6 mice were used. CCRF-CEM cells $\left(3 \times 10^{6}\right)$ were injected i.v. into 5 female NSG mice (The Jackson Laboratory). Seven days later, the mice were treated with TLR1-TLR2 ligand $\left(\mathrm{Pam}_{3} \mathrm{CysK}_{4}, 2 \mathrm{mg} / \mathrm{kg}\right.$ ) or control (PBS) 3 times every other day. On day 14, the number of T-ALL cells in the circulation and BM was determined by staining cells with antihuman CD7 and anti-human CD5 antibodies and analyzing by FACS. To compare T-ALL numbers between mice, calibration microbeads (BD Biosciences - Pharmingen) were added to $50 \mu \mathrm{l}$ blood (or BM or spleen suspensions), and the flow cytometric instrument gates 
were set to count a constant number of beads. In other experiments, mice $(n=5)$ were injected i.p. with IRAK1/4 inhibitor $(10 \mathrm{mg} / \mathrm{kg})$ on days 3, 6, and 9 for mice injected with CCRF-CEM cells, or on days 7,10 , and 13 for mice injected with patient T-ALL cells. The number of T-ALL cells $\left(\mathrm{CD}^{+}\right)$in the BM and blood was determined on day 21 by flow cytometry. In other experiments, patient T-ALL cells were injected into NSG mice, and treatment was initiated after detecting patient T-ALL cells $\left(\mathrm{CD}^{+}\right)$in the blood. Stable Jurkat cell lines with reduced IRAK1 expression were generated using lentiviral vectors expressing IRAK1 shRNA or a vector (selected using puromycin over several weeks) and then injected i.v. into NSG mice. The numbers of T-ALL cells in the BM, blood, and/or spleen were measured by staining cells with anti-human CD7 and/or CD5. For in vivo drug administration, ABT-737 (40 mg/kg), IRAK inhibitor $(10 \mathrm{mg} / \mathrm{kg}$ or $5 \mathrm{mg} / \mathrm{kg}$ when in combination with ABT-737), or vincristine $(0.5 \mathrm{mg} / \mathrm{kg})$ was injected i.p. into NSG mice.

\section{Statistics}

A $P$ value of less than 0.05 was considered statistically significant. All $P$ values were determined using the 2-tailed unpaired $t$ test, with the exceptions noted here. The value in Figure $4 \mathrm{C}$ was determined using multiplemeasures ANOVA. The values for the studies in Figure 4D, Figure 6F, and Figure 9E were determined using the log-rank test with Bonferroni's correction for comparison of multiple between-group differences. Synergy between combination treatments was determined by the combination index using CompuSyn software, available online. In experiments evaluating T-ALL cell counts, the values are shown as the mean \pm SD of 5 mice per group, and for experiments evaluating T-ALL proliferation, the values are shown as the mean \pm SD of triplicate readings.

\section{Study approval}

All tissue collection was performed under protocols approved by the IRB of the University of Maryland Baltimore School of Medicine. All human subjects provided written informed consent prior to their participation in the study. All experiments using mice were approved by the IACUC of the University of Maryland Baltimore School of Medicine.

\section{Acknowledgments}

This work was supported by grants from the Leukemia and Lymphoma Society Translational Research Program, the National Cancer Institute (R01CA140917), and the University of Maryland Greenebaum Cancer Center. The authors thank the Flow Cytometry Shared Services and the Confocal Core Facility of the University of Maryland Greenebaum Cancer Center for cell sorting (FCCF) and for their generous technical support. We thank Sabina Chiaretti of the University of Rome "La Sapienza" for providing the microarray data sets for comparison of IRAK1 mRNA levels between patients with ongoing remission or relapse.

Address correspondence to: Eduardo Davila, University of Maryland Greenebaum Cancer Center, Room 10-041, Bressler Research Building, 655 West Baltimore Street, Baltimore, Maryland 21201-1559, USA. Phone: 410.706.5051; E-mail: EDavila@ som.umaryland.edu.
1. Dores GM, Devesa SS, Curtis RE, Linet MS, Morton LM. Acute leukemia incidence and patient survival among children and adults in the United States, 2001-2007. Blood. 2012;119(1):34-43.

2. Faderl S, et al. Adult acute lymphoblastic leukemia: concepts and strategies. Cancer. 2010;116(5):1165-1176.

3. Pui CH. T cell acute lymphoblastic leukemia: NOTCHing the way toward a better treatment outcome. Cancer Cell. 2009;15(2):85-87.

4. Bartholdy C, Christensen JE, Grujic M, Christensen JP, Thomsen AR. T-cell intrinsic expression of MyD88 is required for sustained expansion of the virus-specific $\mathrm{CD}^{+} \mathrm{T}$-cell population in LCMV-infected mice. J Gen Virol. 2009;90(pt 2):423-431.

5. Geng D, et al. Amplifying TLR-MyD88 signals within tumor-specific $\mathrm{T}$ cells enhances antitumor activity to suboptimal levels of weakly immunogenic tumor antigens. Cancer Res. 2010;70(19):7442-7454.

6. Geng D, Zheng L, Srivastava R, Asprodites N, Velasco-Gonzalez C, Davila E. When Toll-like receptor and $\mathrm{T}$-cell receptor signals collide: $\mathrm{a}$ mechanism for enhanced CD8 T-cell effector function. Blood. 2010;116(18):3494-3504.

7. LaRosa DF, et al. T cell expression of MyD88 is required for resistance to Toxoplasma gondii. Proc Natl Acad Sci U S A. 2008;105(10):3855-3860.

8. Quigley M, Martinez J, Huang X, Yang Y. A critical role for direct TLR2-MyD88 signaling in CD8 T-cell clonal expansion and memory formation following vaccinia viral infection. Blood. 2009;113(10):2256-2264.
9. Rahman AH, et al. MyD88 plays a critical T cellintrinsic role in supporting CD8 T cell expansion during acute lymphocytic choriomeningitis virus infection. J Immunol. 2008;181(6):3804-3810.

10. Zhao Y, De TC, Flynn R, Ware CF, Croft M, Salek-Ardakani S. The adaptor molecule MyD88 directly promotes CD8 T cell responses to vaccinia virus. J Immunol. 2009;182(10):6278-6286

11. Akira S, Hemmi H. Recognition of pathogenassociated molecular patterns by TLR family. Immunol Lett. 2003;85(2):85-95.

12. Cheng $\mathrm{H}$, et al. Regulation of IRAK- 4 kinase activity via autophosphorylation within its activation loop. Biochem Biophys Res Commun. 2007;352(3):609-616.

13. Kollewe C, et al. Sequential autophosphorylation steps in the interleukin-1 receptor-associated kinase-1 regulate its availability as an adapter in interleukin-1 signaling. J Biol Chem . 2004;279(7):5227-5236.

14. Skaug B, Jiang X, Chen ZJ. The role of ubiquitin in $\mathrm{NF}-\kappa \mathrm{B}$ regulatory pathways. Annu Rev Biochem. 2009;78:769-796.

15. Linares JF, Duran A, Yajima T, Pasparakis M, Moscat J, Diaz-Meco MT. K63 polyubiquitination and activation of mTOR by the p62-TRAF6 complex in nutrient-activated cells. Mol Cell. 2013;51(2):283-296.

16. Yang WL, et al. The E3 ligase TRAF6 regulates Akt ubiquitination and activation. Science. 2009;325(5944):1134-1138.

17. $\mathrm{Mu}$ Y, et al. TRAF6 ubiquitinates TGF $\beta$ type I receptor to promote its cleavage and nuclear translocation in cancer. Nat Commun.

\section{1;2:330}

18. Suzuki N, et al. A critical role for the innate immune signaling molecule IRAK- 4 in T cell activation. Science. 2006;311(5769):1927-1932.

19. McDonald DR, et al. Impaired T-cell receptor activation in IL-1 receptor-associated kinase-4deficient patients. JAllergy Clin Immunol. 2010;126(2):332-337.

20. Picard C, et al. Clinical features and outcome of patients with IRAK-4 and MyD88 deficiency. Medicine (Baltimore). 2010;89(6):403-425.

21. Asprodites N, Zheng L, Geng D, Velasco-Gonzalez C, Sanchez-Perez L, Davila E. Engagement of Toll-like receptor-2 on cytotoxic T-lymphocytes occurs in vivo and augments antitumor activity. FASEB J. 2008;22(10):3628-3637.

22. Bendigs S, Salzer U, Lipford GB, Wagner H, Heeg K. CpG-oligodeoxynucleotides co-stimulate primary T cells in the absence of antigen-presenting cells. Eur J Immunol. 1999;29(4):1209-1218.

23. Caron $G$, et al. Direct stimulation of human $T$ cells via TLR5 and TLR7/8: flagellin and R-848 up-regulate proliferation and IFN-gamma production by memory $\mathrm{CD} 4^{+} \mathrm{T}$ cells. J Immunol. 2005;175(3):1551-1557.

24. Cottalorda A, et al. TLR2 engagement on memory CD8(+) T cells improves their cytokine-mediated proliferation and IFN- $\gamma$ secretion in the absence of Ag. Eur J Immunol. 2009;39(10):2673-2681.

25. Komai-Koma M, Jones L, Ogg GS, Xu D, Liew FY. TLR2 is expressed on activated T cells as a costimulatory receptor. Proc Natl Acad Sci US A. 2004;101(9):3029-3034. 
26. Gelman AE, Zhang J, Choi Y, Turka LA. Toll-like receptor ligands directly promote activated $\mathrm{CD} 4^{+} \mathrm{T}$ cell survival. JImmunol. 2004;172(10):6065-6073.

27. Zheng L, Asprodites N, Keene AH, Rodriguez P, Brown KD, Davila E. TLR9 engagement on $\mathrm{CD} 4 \mathrm{~T}$ lymphocytes represses \{gamma\}-radiation-induced apoptosis through activation of checkpoint kinase response elements. Blood. 2008;111(5):2704-2713.

28. Mollaki V, et al. Polymorphisms and haplotypes in TLR9 and MYD88 are associated with the development of Hodgkin's lymphoma: a candidate-gene association study. J Hum Genet. 2009;54(11):655-659.

29. Ngo VN, et al. Oncogenically active MYD88 mutations in human lymphoma. Nature. 2011;470(7332):115-119.

30. Yang G, et al. A mutation in MYD88 (L265P) supports the survival of lymphoplasmacytic cells by activation of Bruton tyrosine kinase in Waldenstrom macroglobulinemia. Blood 2013;122(7):1222-1232.

31. Rhyasen GW, et al. Targeting IRAK1 as a Therapeutic Approach for Myelodysplastic Syndrome. Cancer Cell. 2013;24(1):90-104.

32. Barretina J, et al. The Cancer Cell Line Encyclopedia enables predictive modelling of anticancer drug sensitivity. Nature. 2012;483(7391):603-607.

33. Cao Z, Henzel WJ, Gao X. IRAK: a kinase associated with the interleukin-1 receptor. Science. 1996;271(5252):1128-1131.

34. Li S, Strelow A, Fontana EJ, Wesche H. IRAK-4: a novel member of the IRAK family with the properties of an IRAK-kinase. Proc Natl Acad Sci U S A. 2002;99(8):5567-5572.

35. Wang Z, Wesche H, Stevens T, Walker N, Yeh WC. IRAK-4 inhibitors for inflammation. Curr Top Med Chem. 2009;9(8):724-737.

36. Fitzgerald KA, et al. Mal (MyD88-adapter-like) is required for Toll-like receptor-4 signal transduction. Nature. 2001;413(6851):78-83.

37. Powers JP, et al. Discovery and initial SAR of inhibitors of interleukin-1 receptor-associated kinase-4. Bioorg Med Chem Lett. 2006;16(11):2842-2845.

38. Chiaretti S, et al. Gene expression profile of adult T-cell acute lymphocytic leukemia identifies distinct subsets of patients with different response to therapy and survival. Blood. 2004;103(7):2771-2778

39. Derouet M, Thomas L, Cross A, Moots RJ Edwards SW. Granulocyte macrophage colony-stimulating factor signaling and protea- some inhibition delay neutrophil apoptosis by increasing the stability of Mcl-1.J Biol Chem. 2004;279(26):26915-26921.

40. Henson ES, Gibson EM, Villanueva J, Bristow NA, Haney N, Gibson SB. Increased expression of Mcl- 1 is responsible for the blockage of TRAILinduced apoptosis mediated by EGF/ErbB1 signaling pathway. JCell Biochem. 2003;89(6):1177-1192.

41. Maurer U, Charvet C, Wagman AS, Dejardin E, Green DR. Glycogen synthase kinase-3 regulates mitochondrial outer membrane permeabilization and apoptosis by destabilization of MCL-1. Mol Cell. 2006;21(6):749-760.

42. Chao JR, et al. mcl-1 is an immediate-early gene activated by the granulocyte-macrophage colony-stimulating factor (GM-CSF) signaling pathway and is one component of the GM-CSF viability response. Mol Cell Biol. 1998;18(8):4883-4898.

43. Nijhawan D, et al. Elimination of Mcl-1 is required for the initiation of apoptosis following ultraviolet irradiation. Genes Dev. 2003;17(12):1475-1486

44. Kohn AD, Takeuchi F, Roth RA. Akt, a pleckstrin homology domain containing kinase, is activated primarily by phosphorylation. J Biol Chem. 1996;271(36):21920-21926.

45. Mercurio F, et al. IKK-1 and IKK-2: cytokine-activated IkappaB kinases essential for NF-kappaB activation. Science. 1997;278(5339):860-866.

46. Craig RW. MCL1 provides a window on the role of the BCL2 family in cell proliferation, differentiation and tumorigenesis. Leukemia 2002;16(4):444-454.

47. Konopleva M, et al. MEK inhibition enhances ABT-737-induced leukemia cell apoptosis via prevention of ERK-activated MCL-1 induction and modulation of MCL-1/BIM complex. Leukemia. 2012;26(4):778-787.

48. Weng AP, et al. Activating mutations of NOTCH1 in human $\mathrm{T}$ cell acute lymphoblastic leukemia. Science. 2004;306(5694):269-271.

49. Ferrando AA, et al. Gene expression signatures define novel oncogenic pathways in $\mathrm{T}$ cell acute lymphoblastic leukemia. Cancer Cell. 2002;1(1):75-87.

50. Hebert J, Cayuela JM, Berkeley J, Sigaux F. Candidate tumor-suppressor genes MTS1 (p16INK4A) and MTS2 (p15INK4B) display frequent homozygous deletions in primary cells from $\mathrm{T}$ - but not from B-cell lineage acute lymphoblastic leukemias. Blood. 1994;84(12):4038-4044.

51. Mizobe T, et al. Constitutive association of
MyD88 to IRAK in HTLV-I-transformed T cells. Exp Hematol. 2007;35(12):1812-1822.

52. Matzinger P. Tolerance, danger, and the extended family. Annu Rev Immunol. 1994;12:991-1045.

53. Chen W, Syldath U, Bellmann K, Burkart V, Kolb H. Human 60-kDa heat-shock protein: a danger signal to the innate immune system. J Immunol. 1999;162(6):3212-3219.

54. Wallin RP, Lundqvist A, More SH, von BA, Kiessling R, Ljunggren HG. Heat-shock proteins as activators of the innate immune system. Trends Immunol. 2002;23(3):130-135.

55. Vabulas RM, et al. Endocytosed HSP60s use toll-like receptor 2 (TLR2) and TLR4 to activate the toll/interleukin-1 receptor signaling pathway in innate immune cells. J Biol Chem. 2001;276(33):31332-31339.

56. Wheeler A, Archbold SM, Hardie T, Watson LM. Children with cochlear implants: the communication journey. Cochlear Implants Int 2009;10(1):41-62.

57. van der Heijden IM, et al. Presence of bacterial DNA and bacterial peptidoglycans in joints of patients with rheumatoid arthritis and other arthritides. Arthritis Rheum. 2000;43(3):593-598.

58. Sobek V, et al. Direct Toll-like receptor 2 mediated co-stimulation of $\mathrm{T}$ cells in the mouse system as a basis for chronic inflammatory joint disease. Arthritis Res Ther. 2004;6(5):R433-R446.

59. Smith TJ, et al. Differential expression of Toll-like receptors in follicular lymphoma, diffuse large B-cell lymphoma and peripheral T-cell lymphoma. Exp Mol Pathol. 2010;89(3):284-290.

60. Jarrousse V, Quereux G, Marques-Briand S, Knol AC, Khammari A, Dreno B. Toll-like receptors 2, 4 and 9 expression in cutaneous T-cell lymphoma (mycosis fungoides and Sezary syndrome). Eur $J$ Dermatol.2006;16(6):636-641.

61. Liu H, et al. TRAF6 activation in multiple myeloma: a potential therapeutic target. Clin Lymphoma Myeloma Leuk. 2012;12(3):155-163.

62. Starczynowski DT, et al. TRAF6 is an amplified oncogene bridging the RAS and NF-kappaB pathways in human lung cancer. JClin Invest. 2011;121(10):4095-4105.

63. Sun H, Li XB, Meng Y, Fan L, Li M, Fang J. TRAF6 upregulates expression of HIF-1alpha and promotes tumor angiogenesis. Cancer Res. 2013;73(15):4950-4959.

64. Yeoh EJ, et al. Classification, subtype discovery, and prediction of outcome in pediatric acute lymphoblastic leukemia by gene expression profiling. Cancer Cell. 2002;1(2):133-143. 